\title{
Methods and Devices of Speckle-Noise Suppression (Review)
}

\author{
Igor Kompanets*, Nikolay Zalyapin \\ Quantum Radiophysics Division, P.N. Lebedev Physical Institute, Moscow, Russia \\ Email: *kompan@lebedev.ru
}

How to cite this paper: Kompanets, I. and Zalyapin, N. (2020) Methods and Devices of Speckle-Noise Suppression (Review). Optics and Photonics Journal, 10, 219-250. https://doi.org/10.4236/opj.2020.1010023

Received: October 14, 2020

Accepted: October 28, 2020

Published: October 31, 2020

Copyright $\odot 2020$ by author(s) and Scientific Research Publishing Inc. This work is licensed under the Creative Commons Attribution International License (CC BY 4.0).

http://creativecommons.org/licenses/by/4.0/

\begin{abstract}
Speckles in images formed by a laser beam are interference noise arising due to coherent nature of laser radiation. As the number of projection systems using laser light sources increases, new methods and devices appear for reducing the contrast of speckle-noise and thereby suppressing it. Taking into account the long-standing nature of the problem and the continuing relevance of its solution, this review discusses modern practical methods and devices-despecklers, used to reduce the speckle contrast and to achieve high quality of projected images. The review discusses the nature of the speckles appearance, considers their statistical properties, and describes the measurement of the speckle contrast. The requirements for despecklers are considered, the most important of which are speckle suppression efficiency, simplicity of design and compactness, low power consumption and low optical loss. The characteristics of different types of despecklers, including diffusers, devices based on electroactive polymers, optical waveguides, colloidal solutions and liquid crystals, as well as orthogonal phase matrices and diffraction gratings proposed as despeckers, are examined in detail. It is shown that despecklers with decorrelation of the phase front based on the mirror deformation and optical fiber have less light losses. Electro-optical-liquid crystal despecklers do not have mechanically deformable or moving elements that reduce the reliability and durability of operation, and are more compact and simple in design. A comparative table of characteristics of the most effective despecklers in which the speckle contrast is reduced to ten percent or less, with an indication of their advantages and disadvantages, is given.
\end{abstract}

\section{Keywords}

Projection System, Laser Beam, Speckle-Noise, Speckle Suppression

\section{Introduction}

Despite the obvious advantages of laser light sources (wide color spectrum, high 
brightness, long lifetime, directivity, high optical power, etc.) in comparison with traditional lamps and light-emitting diodes (LEDs), the presence of light spots-speckles in the image generated by a laser prevents widespread use of lasers in projection systems. Speckles are interference noise and arise due to the coherent nature of laser radiation [1]. Speckle-noise reduces image quality and the observer's ability to see fine details in images.

Speckle-noise in the observed images can be reduced by averaging it directly on the screen or by destroying the phase relationships in the laser beam, that is, before projecting the images onto the screen. The averaging of the speckle structure on the screen is achieved using a rapidly moving (for example, rapidly rotating) light-scattering diffuser [2]. In the known development of a holographic memory [3], more uniform holographic images were observed when two diffusers moved relative to each other.

It is clear that a screen with mechanical movement is not the best solution of the problem. The speckle suppression device-despeckler (Figure 1(a)), based on the destruction of phase relationships in the laser beam, seems to be more compact and efficient, but it should have a much higher resolution-on the order of hundreds and even thousands of $1 / \mathrm{mm}$ (due to the need for subsequent beam expansion to screen size), and should not degrade the intensity of the laser beam and its directivity. Such a device was originally [4] a transparent phase mask with a random spatial distribution of the phase with a depth of the order of $\pi$, realized using a rapidly rotating bleached photographic plate previously exposed through a diffuser (usually frosted glass).

Many other methods and devices for suppressing speckle structures are known as well that have appeared in recent years. Taking into account the continuing urgency of the problem, below a review of modern practical methods and devices is presented for effectively reducing speckle contrast in a laser image and thereby suppressing speckle-noise in it.

Experiments have shown that a despeckler can be recognized as effective, which is capable of providing a speckle pattern contrast of less than $3 \%$ for still images [5] and no more than 5\% for moving images [6]. In this case the observer does not notice the noise practically [7]. However, we will consider below the methods and devices providing the value of speckle contrast at the level of about $10 \%$, when the quality of the generated image is quite acceptable. This will allow taking into account different (sometimes unknown) settings of measurement parameters used in different devices. Besides, some methods can be still improved or be of interest and unordinary for readers. A qualitative comparative assessment of considered methods and despecklers will be given also.

\section{Nature and Measurements of Speckles}

\subsection{Formation of Speckle Structures}

Laser radiation has a high degree of temporal coherence (monochromaticity) and spatial coherence [8] [9]. When it illuminates randomly inhomogeneous 


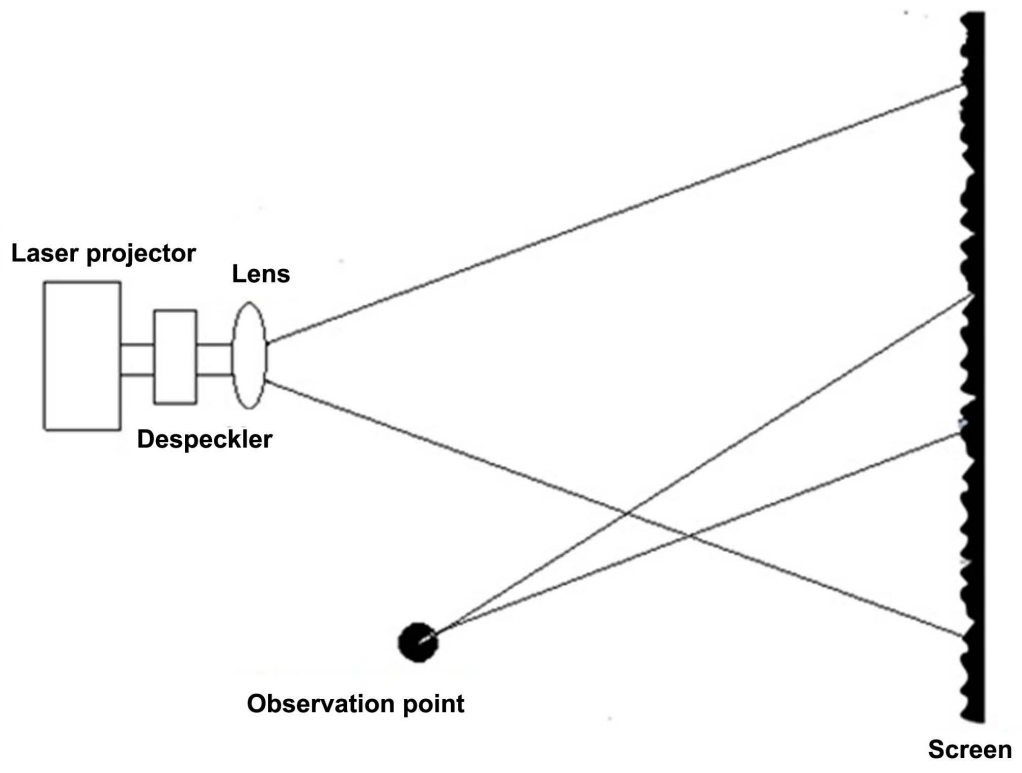

(a)

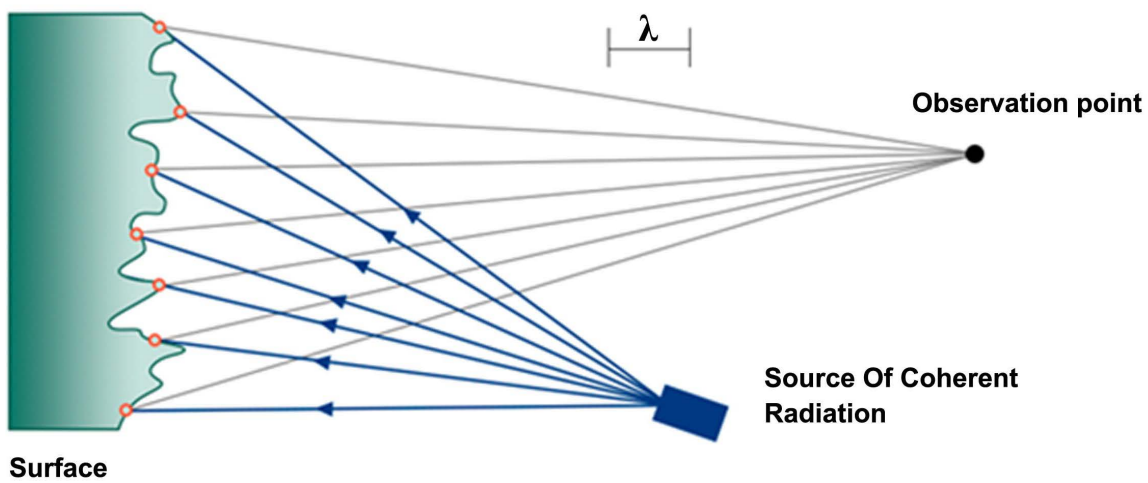

(b)

Figure 1. General scheme of formation and registration of interference speckle structures in the image formed by a laser projector (a) and a simplified scheme of their observation on the screen (b).

objects, such as, for example, a rough surface or a transparent medium with a refractive index varying in space, as a result of the interference of laser beams, the intensity of the reflected or transmitted wave is characterized by large spatial fluctuations due to the overlap of many out-of-phase scattering or blurring functions, each of which corresponds to its point (its heterogeneity) of the object.

In Figure 1(b) by the example of a rough surface a diagram of the formation and observation (registration) of such fluctuations is showed. They lead to the formation of a complex spatially spotty structure of the light field-a speckle structure and, accordingly, to the formation of a speckle pattern in the image, which is, in fact, speckle-noise in it.

The resulting field amplitude (Figure 1(b)) at an arbitrary observation point $P_{0}$, taking into account the contribution of phase shifts $\Delta \varphi_{n}$ and the amplitudes 
$a_{n}$ of each elementary wave scattered by surface or medium inhomogeneities, is determined as

$$
U\left(P_{0}\right)=\sum_{n=1}^{N} a_{n} \exp \left(i \Delta \varphi_{n}\right)=A\left(P_{0}\right) \exp \left(i \varphi\left(P_{0}\right)\right),
$$

where $N$ is the total number of independent sources of secondary waves on the illuminated area of a surface [10].

In this case, if the roughness or spatial inhomogeneities have a random character over the area of the object, then the resulting interference spots will be located randomly in the observation plane also (Figure 2). If the surface of an object is rough on the scale of optical wavelengths (a common situation in practice), then the image looks grainy, with many light and dark spots that have no visible connection with the macroscopic scattering properties of the object [11], [12]. Contrast pronounced speckle structures are observed when phase shifts are in the range of $0 \ldots 2 \pi$ radians. It is sufficient for this, for example, that the heights of the surface inhomogeneities are comparable to the wavelength of the incident light.

Thus, a speckle structure is a random interference pattern, which is formed due to mutual interference of coherent waves with a random set of intensities and/or random phase shifts [10].

\subsection{Statistical Properties of Speckles}

An aggregate of a large number of elementary light sources having phases and amplitudes statistically independent of each other is a diffuse scattering source. It is assumed that the illuminated section of the diffuse scatterer is much larger than the area of an individual elementary source, and the phases are uniformly distributed in the interval $(-\pi \ldots+\pi)$. Speckle fields formed during scattering of radiation on such diffuse scattering sources are called developed speckle fields, and speckle structure is called developed speckle structure.

Since we do not know the detailed microscopic structure of a complex wave front leaving a scattering object, we use statistical approach to the question of the speckle structure properties. One of the important statistical characteristics

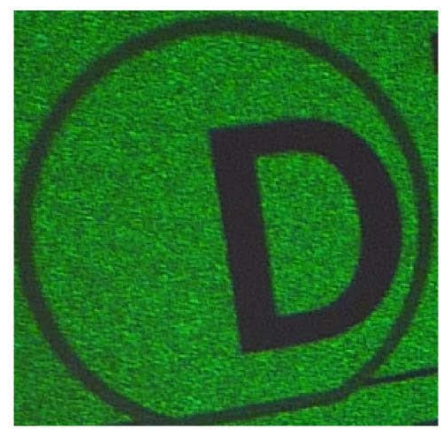

(a)

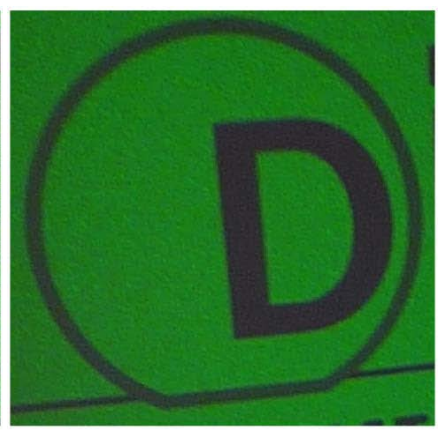

(b)

Figure 2. An example of an image in the presence (a) and in the absence (b) of speckle structures as interference speckle-noise (Ref. [13], Figure 10). 
of the speckle structure is the intensity distribution density I in a certain point in the image.

In many fields of physics, and in optics in particular, one has to deal with complex random variables, which are the sum of many small "elementary" complex contributions. The role of such complex numbers is often played by the so-called phasors [12], which characterize the amplitude and phase of the disturbance of a monochromatic or quasi-monochromatic wave.

The total complex wave amplitude (resulting phasor), formed due to scattering on a set of small independent scatterers, is the sum of random phasors and is defined as:

$$
A=A e^{i \theta}=\frac{1}{\sqrt{N}} \sum_{n=1}^{N} a_{n} e^{i \varphi_{n}}
$$

where $a_{n}$ is the amplitude, and $\varphi_{n}$ are the phases of each elementary wave, $N$ is the number of complex contributions (complex phasors).

To simplify the analysis, the following assumptions are made [14]:

- The amplitude $a_{n}$ and the phase $\varphi_{n}$ of a specific elementary wave are statistically independent of each other.

- The amplitude $a_{n}$ and the phase $\varphi_{n}$ of any elementary wave are statistically independent of the amplitudes and phases of all other elementary waves (uncorrelated).

- The phases are evenly distributed in the interval $(-\pi, \pi)$.

The intensity $I$ and the amplitude $A$ are related as follows:

$$
I=A^{2}
$$

when $N$ is infinite, the joint probability density function of amplitude and phase can be expressed as:

$$
P_{A, \theta}(A, \theta)=\frac{A}{2 \pi \sigma^{2}} \exp \left(-\frac{A^{2}}{2 \sigma^{2}}\right) .
$$

Then the probability density function of the amplitude is the Rayleigh distribution:

$$
P_{A}(A)=\frac{A}{\sigma^{2}} \exp \left(-\frac{A^{2}}{2 \sigma^{2}}\right)
$$

Considering that

$$
P_{I}(I)=P_{A}(\sqrt{I})\left|\frac{\mathrm{d} A}{\mathrm{~d} I}\right|=\frac{1}{2 \sqrt{I}} P_{A}(\sqrt{I}),
$$

the corresponding probability density function of the intensity for $I>0$ can be represented as:

$$
P_{I}(I)=\frac{\sqrt{I}}{\sigma^{2}} \exp \left(-\frac{I}{2 \sigma^{2}}\right) \frac{1}{2 \sqrt{I}}=\frac{I}{2 \sigma^{2}} \exp \left(-\frac{I}{2 \sigma^{2}}\right)
$$

The values of this distribution are easy to find by direct integration, and a result is 


$$
\overline{I^{q}}=\left(2 \sigma^{2}\right)^{q} q !
$$

It can be seen that the average intensity $\bar{I}$ is equal to $2 \sigma_{I}$. Therefore, the equivalent statement is that the $q$-th result is defined as follows:

$$
\overline{I^{q}}=\bar{I}^{q} q !,
$$

and the probability density function can be rewritten as:

$$
P_{I}(I)=\left(\frac{1}{\bar{I}}\right) \exp \left(-\frac{I}{\bar{I}}\right) .
$$

Speckles with such intensity distribution are called fully developed speckles. Dispersion and standard deviation of the intensity for the current case are determined as

$$
\overline{I^{2}}=2 \bar{I}^{2}, \quad \sigma_{I}^{2}=2 \bar{I}^{2}, \sigma_{I}=\bar{I} .
$$

An important characteristic of speckles, characterizing the degree of noise in a speckle pattern (speckle structure), is speckle contrast, which is defined as the ratio of the standard deviation of intensity fluctuation to the mean value of the intensity $C=\sigma /<I\rangle[1]$. Differently,

$$
C=\frac{\sigma_{I}}{\bar{I}},
$$

that is, the speckle contrast characterizes the depth of spatial modulation of the scattered radiation and shows how strong the intensity fluctuations in the speckle sample are compared to the average intensity.

In this case, the signal-to-noise ratio is the reciprocal value of the speckle contrast and is defined as:

$$
\frac{S}{N}=\frac{1}{C}=\frac{\bar{I}}{\sigma_{I}} .
$$

Speckle contrast values range from 0 to 1 (or from $0 \%$ to $100 \%$ ). Therefore, at $C=1$, the intensity fluctuations of the fully developed speckles are of the same order of magnitude as the average value. In practice, developed speckle fields with the maximum possible value of speckle contrast are observed when wide laser beams are diffracted on a highly rough surface or a strongly scattering transparency [10]. For fully developed speckles, the signal-to-noise ratio is also 1.

\subsection{Measurement of Speckle-Noise}

Depending on the optical scheme for observing speckle patterns, objective and subjective speckles are distinguished [10]. An objective speckle pattern is formed in free space and can be recorded if a photographic film or the CCD matrix itself (without a lens) is placed in the observation plane. In this case, the formed speckle pattern depends on the geometry of the scheme and the coherent radiation wavelength. The diagram of the formation of an objective spectrum pattern is shown in Figure 3(a). 


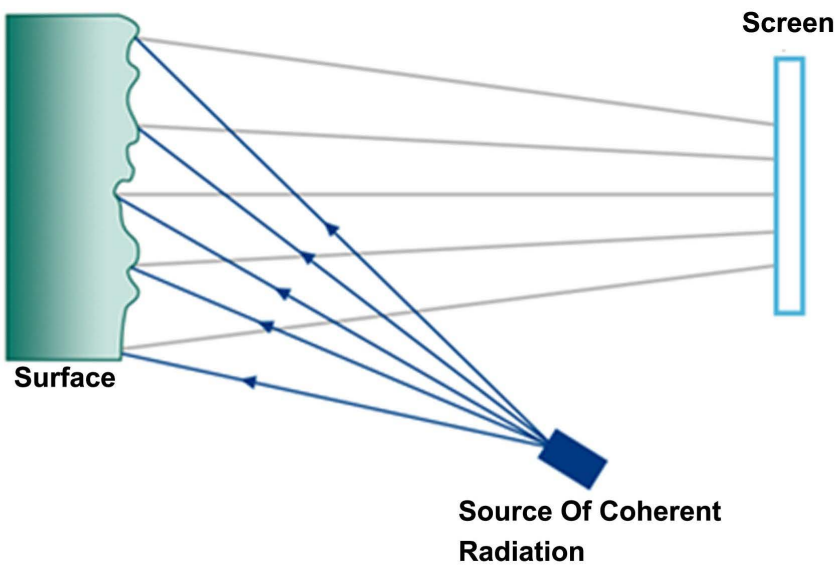

(a)

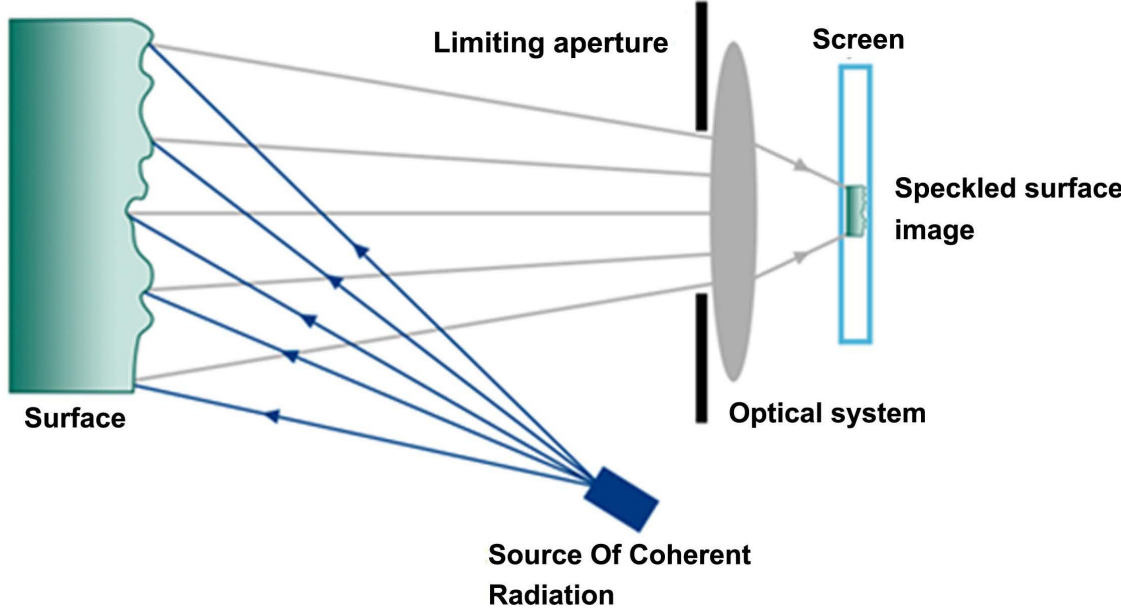

(b)

Figure 3. Optical schemes for the formation of an objective speckle pattern (a) and subjective (b).

A subjective speckle pattern occurs when a scattering surface is displayed on a screen due to an optical system. Thus, if we photograph a speckle picture with a camera, we will get a subjective picture, since its parameters will already depend on the lens of the camera. In this case, the detailed structure of the subjective speckle pattern depends on the parameters of the imaging optical system and its location. The optical scheme of the formation of a subjective spectrum pattern is shown in Figure 3(b).

When the same CCD is used, and a size of objective and subjective speckles is the same, the speckle contrast measured in the image plane, is in good agreement with that when measured in free space [7] [15].

\subsection{Methods for the Formation of Uncorrelated Speckle Structures}

If a rough surface is illuminated by coherent light, the intensity distribution in 
the resulting speckle pattern depends on the properties of the rough surface, the direction of incidence and observation of the laser beam, the temporal and spatial coherence of radiation, as well as on the polarization and phase distribution of the light wave field scattered by the object [16]. If at least one of the parameters changes instantly or sequentially, the speckle-noise can be reduced.

For $M$ uncorrelated speckle structures, the contrast of the resulting speckle pattern can be reduced in accordance with the law $C=\sigma /\langle I\rangle=1 / \sqrt{M}$ [1], and uncorrelated speckle structures can be obtained from a given object by means of selection on time, space, frequency or polarization. Table 1 shows various methods of speckle reduction (suppression) through the instantaneous and sequential formation of uncorrelated speckle structures.

When creating time-varying independent speckle patterns, one should take into account the integration time of the human eye, which is about $30 \mathrm{~ms}$. During measuring speckles, the camera integration time should be comparable to the eye integration time.

\section{Methods and Devices for Suppressing Speckles}

\subsection{Despecklers of Electromechanical Type}

\subsubsection{Moving Diffusers}

Consecutive uncorrelated speckle patterns can be created by a moving (often rotating) diffuser-scatterer through which a laser beam passes. This is the earliest and most widely used speckle suppression method. Such diffusers can generate many uncorrelated speckle patterns. Reduction of contrast and suppression of speckles is achieved in them by averaging speckle structures temporarily.

In 2013, a despeckler was announced, which was an optically rough diffuser with a certain scattering angle [18]. It rotated, and due to the different areas of the diffuser exposed to illumination, different phase functions were superimposed on the light beam. The higher the roughness of the diffuser or the higher

Table 1. Methods for the formation of uncorrelated speckle structures [7] [17].

\begin{tabular}{|c|c|c|}
\hline Decorrelation object & Instant averaging & Sequential averaging \\
\hline Temporal coherence & $\begin{array}{l}\text { Broadband light source; } \\
\text { Many light sources of different wavelengths }\end{array}$ & Dynamic wavelength variation (e.g. tunable laser) \\
\hline Spatial coherence & $\begin{array}{l}\text { Using a spatially expanded light source; } \\
\text { Dividing a light source into spatially incoherent sub-sources by } \\
\text { breaking temporal coherence; } \\
\text { Simultaneous illumination of different areas of the object with } \\
\text { several incoherent light sources }\end{array}$ & Movement (scanning) of a coherent light spot \\
\hline Wave field direction & $\begin{array}{l}\text { Simultaneous illumination with multiple light sources of different } \\
\text { directions }\end{array}$ & Dynamic changing the angular spectrum \\
\hline Polarization & $\begin{array}{l}\text { Splitting a light source into two temporary incoherent tracks with } \\
\text { linear polarization changes }\end{array}$ & Dynamic changing the state of polarization \\
\hline Phase front & & $\begin{array}{l}\text { Dynamic diffuser; } \\
\text { Vibrating multimode fiber }\end{array}$ \\
\hline
\end{tabular}


the rotation speed, the greater the temporary degree of freedom M. By choosing a suitable diffuser scattering angle, the speckle contrast was reduced to $4 \%$.

In [19], a rectangular homogenizing light tube was proposed in the path of the illuminating optics of the projector, which, like a rotating diffuser, rotates to create a variety of angles. Thus, it was possible to achieve a speckle contrast $7.5 \%$.

A despeckler similar in principle [20] consists of two diffusers rotating in opposite directions with different angular velocities, and a spatial filter limiting opening the cone of scattering and speckle decorrelation.

Power Technology, Inc. [21] also offers on a market the simple "IQ7 laser speckle reducer" in the form of a rather long tube (Figure 4(a)), in which a homogeneous diffuser is placed that scatters the beam into an angle of $1^{\circ}, 10^{\circ}$ or $20^{\circ}$ for different applications. Speckle suppression is available in the wavelength range from 405 to $808 \mathrm{~nm}$.

Suppression of speckles in such systems is accompanied with a deterioration in the quality of the light beam and an increase in its divergence. In addition, purely mechanically moving devices are less reliable and durable, and are difficult to be compact.

A vibrating diffuser is more compact (Figure 4(b)). It is located in the intermediate plane of the image and vibrates in a purely sinusoidal mode with a frequency of about $100 \mathrm{~Hz}$ and an amplitude of about $60 \mu \mathrm{m}$ [22]. As shown theoretically, in this mode, the temporal degree of freedom $\mathrm{M}$ becomes infinity, and a low speckle contrast is achieved $(C=3.4 \%)$. However, if the diffuser has non-sinusoidal movement or is not positioned exactly in the intermediate plane, the speckle suppression performance is significantly degraded.

\subsubsection{Electroactive Polymers}

The company Optotune (Switzerland) proposed a speckle suppression method [23], brought to commercial use in the form of the LSR (The Laser Speckle Reducer) despeckler (Figure 5). It uses a diffuser that is periodically displaced in space for a short distance.

The diffuser is mounted on an elastic membrane made of electroactive polymer, which contracts and thickens, depending on the presence of voltage on the electrically conductive coatings attached to it, and thereby moves the diffuser

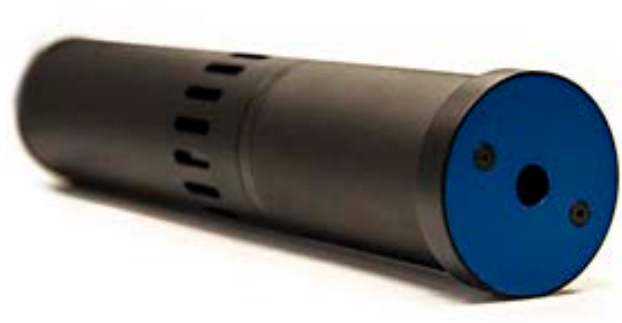

(a)

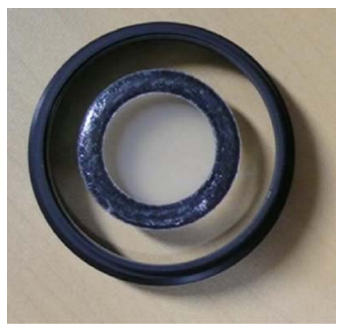

(b)

Figure 4. (a) Despeckler by power technology, Inc. with a homogeneous diffuser [21]; (b) Despeckler with a vibrating diffuser (Ref. [22], Figure 2). 


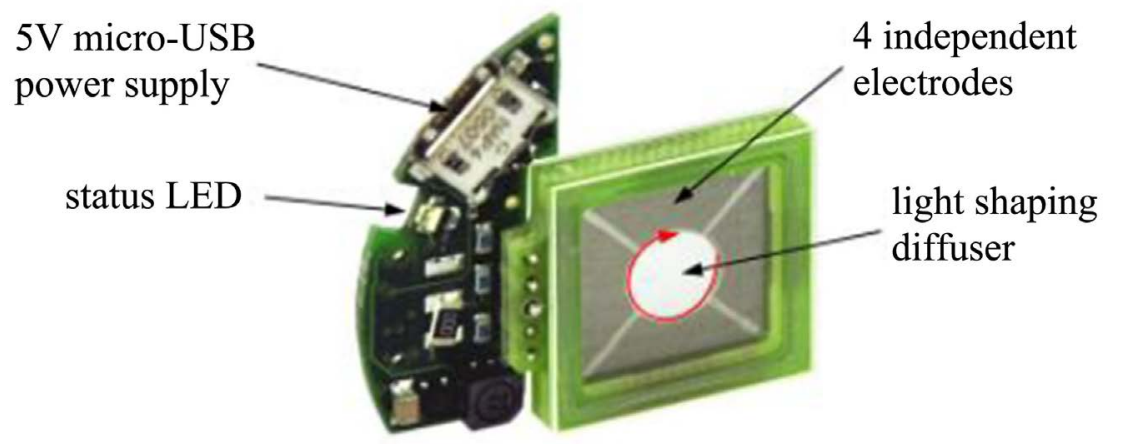

Figure 5. Despeckler LSR-5-17 from optotune (Ref. [23], Figure 1).

forth and back. A different speckle pattern is generated for each individual diffuser position. During the integration time, the superposition of speckle structures results in homogenization of the observed light pattern [15].

Electroactive polymers are known as "artificial muscles". Flexible electrically conductive surfaces are attached to the top and bottom of a thin elastomeric film. When voltage is applied to the electrodes, they both attract each other and thus compress the elastic film (Figure 6).

The maximum reduction in speckle contrast achieved with an LSR is determined by its optical system. The greatest reduction occurs at high frequencies and high vibration amplitudes, in high-resolution diffuser samples and at long integration times. As in all methods with diffusers, the increase in the divergence of the laser beam should be within acceptable limits, and this increase is more significant for very shallow diffuser structures. The efficiency of LSR application increases with decreasing a diffuser surface roughness, since the smaller the grain size, the more completely uncorrelated images are within the diffuser swing. Unfortunately, as the roughness size decreases, the laser radiation divergence increases.

Table 2 shows the characteristics of Optotune LSR despeckers [24]. For LSR-5-17 with an oscillation frequency of $280 \mathrm{~Hz}$, an amplitude of $600 \mu \mathrm{m}$, a divergence of $20^{\circ}$, a roughness size of about $9 \mu \mathrm{m}$, and an integration time of 10 $\mathrm{ms}$, the speckle contrast value was no more than $2 \%$ [23].

\subsection{Fiber Based Despecklers}

\subsubsection{Using the Multimode Optical Fiber and Piezoelectric Vibrator}

One of the effective ways to obtain time-consistent uncorrelated speckle structures is the use of multimode optical fibers. The superposition of contributions from different fiber modes on the output surface forms a speckle pattern that can change over time as a result of fiber bending or vibration. The paper [25] describes a technique for reducing speckle contrast in multimode fibers using a piezoelectric vibrator (Figure 7) and investigates factors affecting the speckle contrast: the diameter and shape of the fiber core, fiber bend radius, vibration frequency, time of speckle pattern registration by a camera, etc.

Table 3 summarizes the characteristics of the multimode fibers used for this 


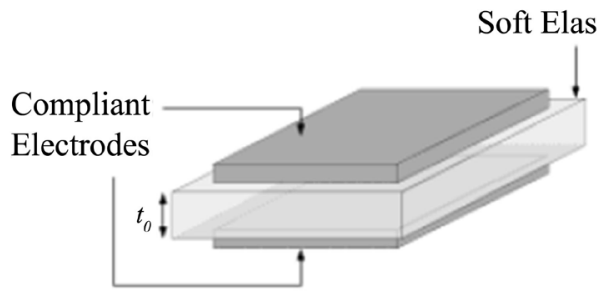

(a)

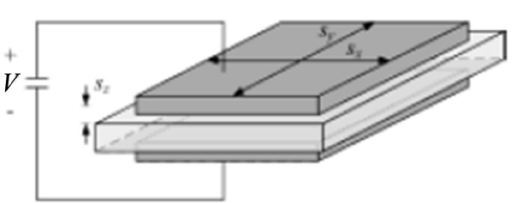

(b)

Figure 6. Electroactive polymer in the absence (a) and in the presence (b) of an electric voltage at the electrodes (Ref. [23], Figure 3).

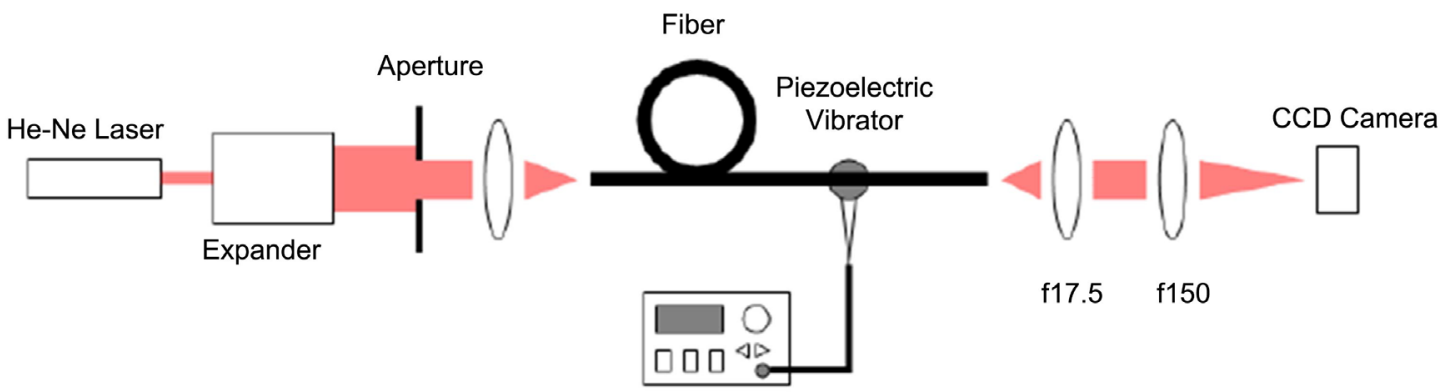

Multi Function Generator

Figure 7. Scheme of despeckler operation based on multimode optical fiber and piezoelectric vibrator (Ref. [25], Figure 1).

Table 2. The main characteristics of the despeckler LSR-5-17 [24].

\begin{tabular}{cc}
\hline Dimensions, $\mathrm{mm}$ & $17 \times 17 \times 3.8$ \\
\hline Weight (only LSR/with electronics), g & $1.44 / 2.55$ \\
Power supply unit (micro-USB interface), $\mathrm{V}_{\mathrm{DC}}, \mathrm{V}$ & 5 \\
Power consumption (with standard electronics), $\mathrm{mW}$ & 310 \\
Electrode capacity, $\mathrm{pF}$ & 75 \\
Working temperature, ${ }^{\circ} \mathrm{C}$ & $-30 \ldots+85$ \\
Speckle structure contrast, C, \% & 2 \\
\hline
\end{tabular}

Table 3. Characteristics of the used multimode fibers [25].

\begin{tabular}{ccccc}
\hline Fiber & Core size, $\mu \mathrm{m}$ & Cladding diameter, $\mu \mathrm{m}$ & NA & Fiber type \\
\hline A & 115 & 200 & 0.22 & Silica core/Silica cladding \\
B & 210 & 300 & 0.57 & Silica core/Polymer cladding \\
C & 250 & 350 & 0.57 & $\begin{array}{c}\text { Heptagonal core } \\
\text { Silica core/Polymer cladding } \\
\text { D }\end{array}$ \\
\hline
\end{tabular}

study. Fibers A, B, and C had a round core, while the fiber C had a polyhedral core. The size of the 7 -angles fiber core $C$ was measured through the diameter of the inserted circle.

For fiber A, the dependences of speckle structures on the vibration frequency, 
as well as on the conditions of the laser beam incidence and the fiber bending radius, were investigated. Figure 8 shows the dependence of the speckle contrast on the vibration frequency. The excitation frequency was varied from 0 to 23 $\mathrm{kHz}$. The highest efficiency (about 14\% and 10\%) of speckle suppression was achieved at frequencies of 3 and $20 \mathrm{kHz}$, which corresponds to the resonant frequency of the basic and higher orders, respectively.

The efficiency of reducing the speckle contrast using a multimode fiber depends on the mode of the light beam propagation in it. There were two types of modes: meridional and oblique. The meridional modes in a fiber are associated with the passage through its central axis. The oblique modes propagate through the fiber on helical trajectory, without passing through its central axis. Accordingly, both modes of rays propagation were considered: through the center of the fiber (meridional modes) and with an offset of $50 \mu \mathrm{m}$ from the center of the core (oblique modes).

Figure 9(d) and Figure 9(e) show speckle patterns and linear intensity profiles in them with vibration switched on for the conditions of the beam propagation through the center of the fiber and with its displacement, respectively. The vibration frequency was set at $20 \mathrm{kHz}$. Speckle-noise was more efficiently suppressed when the beam was displaced from the center as compared to the condition of its incidence into the center of the fiber.

Figure 9(c) and Figure 9(f) show speckle patterns and linear intensity profiles in them under the condition of light beam incidence into the center of the bent fiber with vibration on and off, respectively. The minimum speckle contrast achieved in this experiment was 0.091 .

The efficiency of speckle-noise suppression was also evaluated for various types of fibers, the results of which are shown in Table 3.

Figure 10 shows speckle patterns and linear intensity profiles in them for

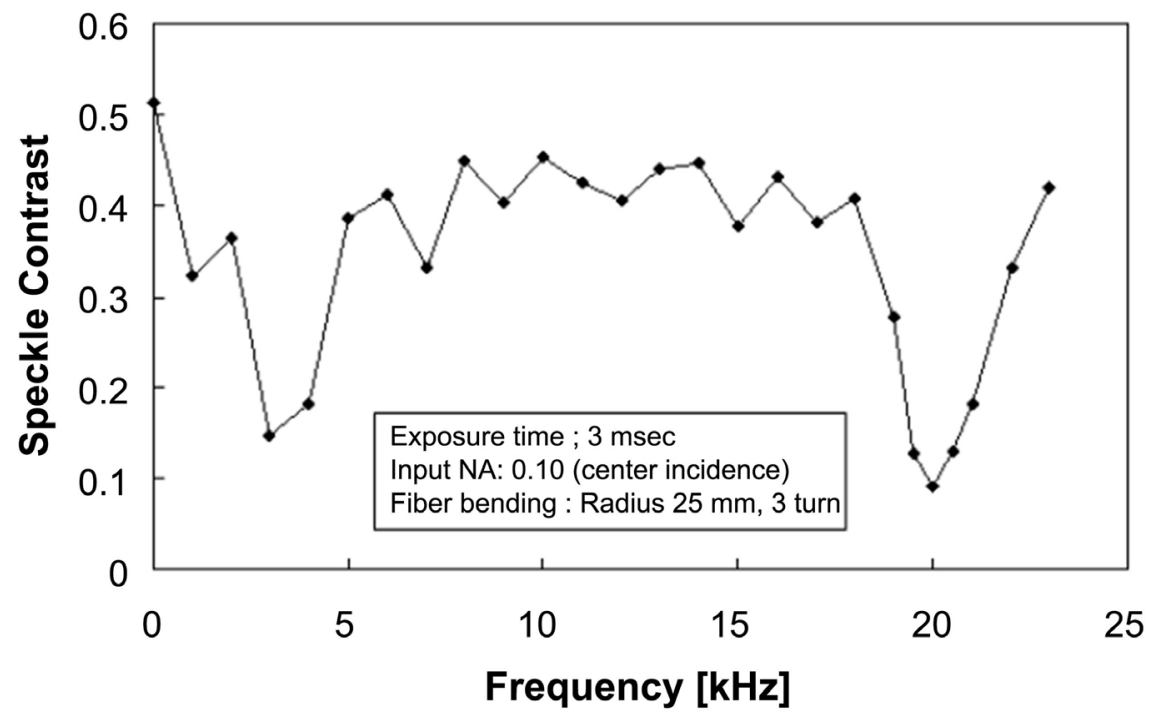

Figure 8. Dependence of speckle contrast on vibration frequency (Ref. [25], Figure 3). 


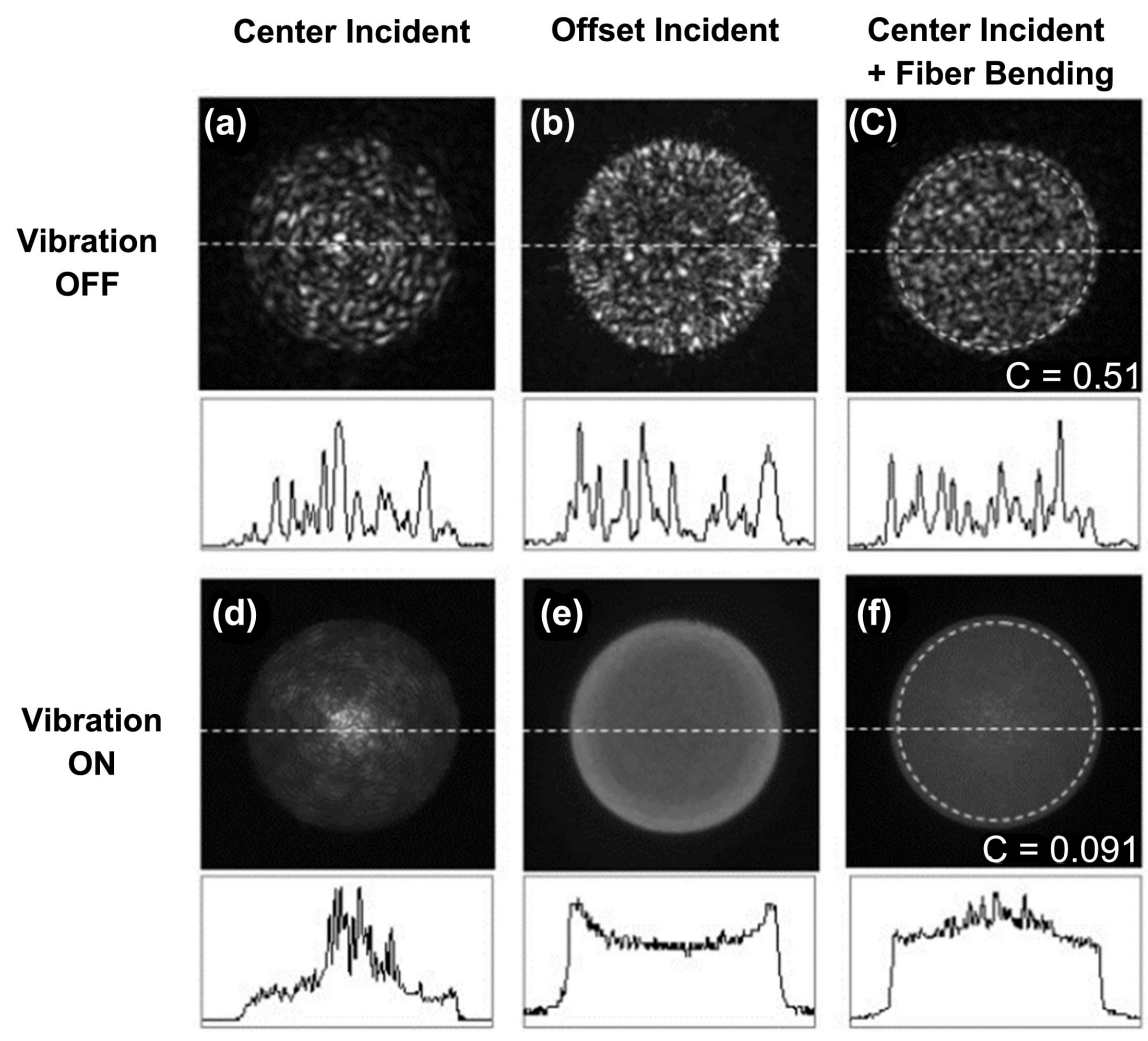

Figure 9. Speckle patterns of the near field and linear intensity profiles in them for fiber A [25]. The profiles are highlighted within a circle framed by white dashed lines. Speckle contrasts are calculated within this circle (Ref. [25], Figure 2).

various types of fibers. Near-field patterns were observed for the multimode fiber B with a core diameter of $210 \mu \mathrm{m}$ and a numerical aperture $\mathrm{NA}=0.57$. For the input of the laser beam, the NA value was 0.10 .

Fiber B, like A, had a round core and was wound into 1 ring with a bend radius of $35 \mathrm{~mm}$. The observed diagrams for vibration off and on conditions are shown in Figure 10(a) and Figure 10(d), respectively. Speckle-noise is well suppressed by vibration, since an almost flat profile surface is obtained. Like the fiber A, the near-field speckle pattern had a flat top profile only when bending was applied to the fiber.

For multimode fiber $\mathrm{C}$ with a heptagonal core, the observed patterns and intensity linear profiles for vibration off and on are shown in Figure 10(b) and Figure 10(e), respectively. Speckle-noise is well suppressed by vibration. Unlike the fibers $\mathrm{A}$ and $\mathrm{B}$ with a round core, a flat top profile was obtained without bending fiber $\mathrm{C}$. The same effect can be expected in other fibers with a non-circular core, such as square core, rectangular core, pentagonal core, hexagonal core, etc.

Figure 10(c) and Figure 10(f) show the output diagrams of a plastic optical fiber without vibration and with vibration, respectively. Speckle-noise was effectively suppressed by vibrating the fiber.

Although plastic fiber has a high speckle reduction ability, it is not suitable for 
Fiber B

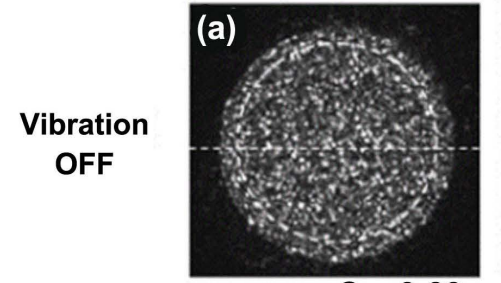

$C=0.60$
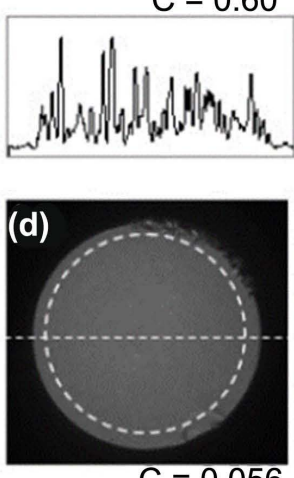

$C=0.056$

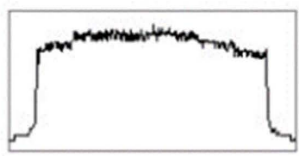

Fiber C

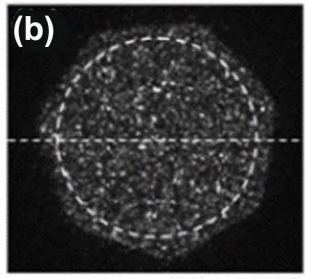

$C=0.60$
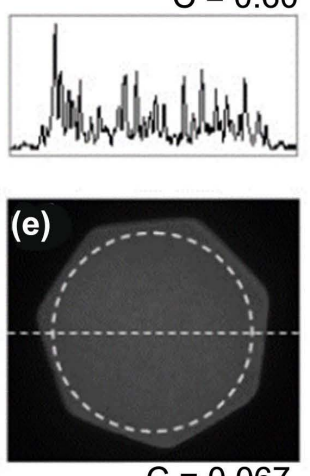

$C=0.067$

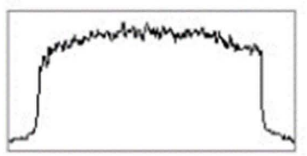

Fiber D

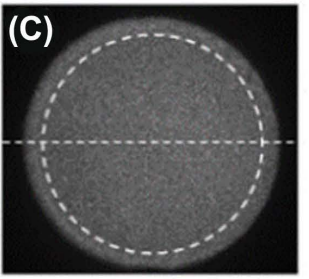

$C=0.42$
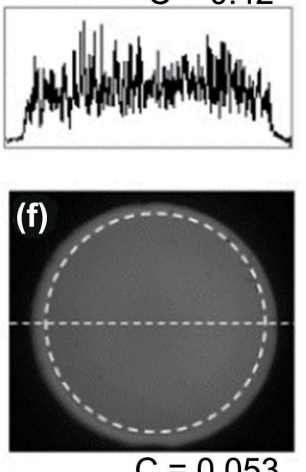

$\mathrm{C}=0.053$

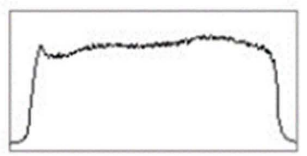

Figure 10. Output speckle patterns and linear intensity profiles in them for different types of fibers. Profiles are highlighted within a circle framed with white dashed lines. Speckle contrasts are calculated within this circle (Ref. [25], Figure 5).

the purpose of effective reducing the speckle contrast. The first reason is the large numerical aperture. Due to the high sensitivity of such fibers to stresses, modes were mixed in them, and the numerical aperture of the output beam became almost equal to the numerical aperture of the fiber. However, higher output numerical aperture requires higher NA optics, which is more complex and expensive. The second reason is the high losses in optical signal transmission in a plastic fiber, much higher than in a quartz fiber. There is also a third reason, which is that, a plastic fiber can only be used at low laser power because its heating and low thermal stability.

The speckle contrast value obtained using the round fiber was 0.056 , and using the heptagonal core fiber was 0.067 . However, in opinion of the authors, a fiber with a non-circular core shape can be more effective in reducing speckle contrast, since it does not require the use of the fiber bending effect and, thereby, allows creating a more compact despeckler.

It should be noted that often even small turbulences in the air surrounding the fiber, or insignificant changes in its temperature, are enough to change the speckle pattern on the output surface.

\subsubsection{Suppression of Speckle-Noise Using the Multimode Fiber and Macrofiber Composite}

In [26], a speckle-noise suppression method was proposed using a flexible and 
lightweight laser module based on a combination of multimode fiber (MMF) and macrofiber composite (MFC). For the experiment, two types of fiber were selected with the characteristics shown in Table 4, as well as a macro-fiber composite (Figure 11) manufactured by Smart Material Corp. (type: M8514-P2) serving as a driver for the MMF vibration.

Macrofiber composite (Figure 11) consists of three main components: piezoceramic rods made of lead zirconate titanate, a pair of thin polyimide films (top and bottom) with etched conducting electrodes, and two layers of adhesive matrix material.

In order to increase damage resistance and flexibility with respect to monolithic ceramics, aligned rectangular piezoceramic rods (bonded with a matrix adhesive) are sandwiched between layers of an adhesive matrix material and a polyimide film. The electrodes are attached to a film that transfers the applied voltage directly to the strip rods. The adhesive binds the drive components together. When voltage is applied to the electrodes of the device, the device is compressed due to the piezoelectric effect. The MFC generates vibrations as a result of the periodic contractions and stretches.

The experimental setup with a low coherent light source and using a combination of MMF and MFC is shown in Figure 12. A laser diode with a center wavelength of $520 \mathrm{~nm}$ is used as a source of laser radiation. Behind it, a linear polarizer for adjusting the light intensity is placed. The laser beam inputs into the MMF through a fiber optic connector with a glass lens of $4.6 \mathrm{~mm}$ focal length. A diffuser is installed just after the MMF exits for additional light scattering.

Table 4. Specifications for two types of multimode fiber (MMF) with different numerical aperture (NA) [26].

\begin{tabular}{cccccc}
\hline Fiber & Length, $\mathrm{m}$ & Core diameter, $\mu \mathrm{m}$ & Fiber diameter, $\mu \mathrm{m}$ & $\mathrm{NA}$ & $\begin{array}{c}\text { Refractive index of } \\
\text { core/cladding }\end{array}$ \\
\hline $\mathrm{A}$ & 0.4 & 1470 & 1500 & 0.51 & $1.492 / 1.402$ \\
$\mathrm{~B}$ & 0.4 & 400 & 970 & 0.22 & $1.457 / 1.439$ \\
\hline
\end{tabular}

(a)
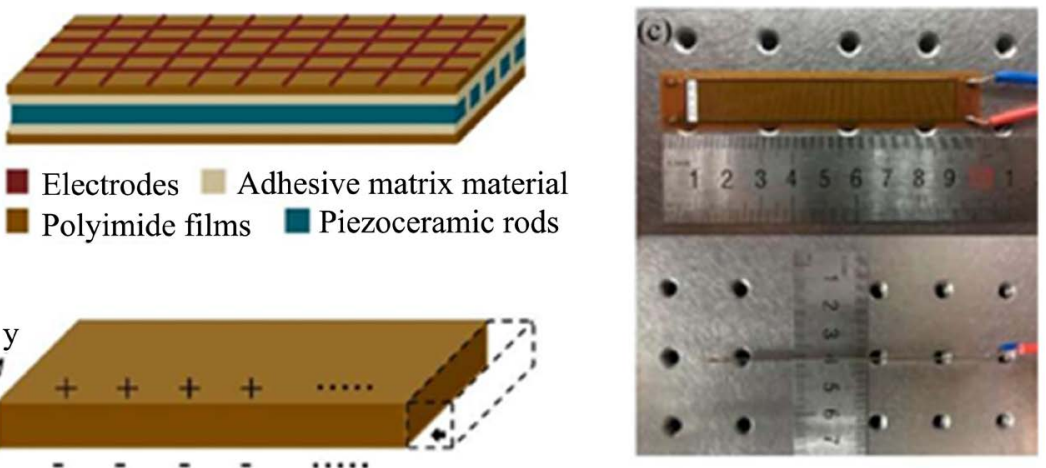

(b)

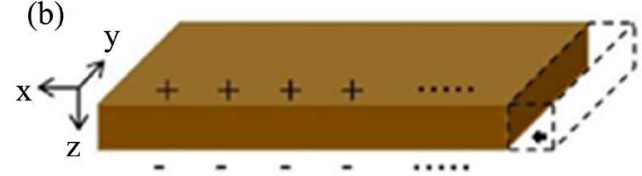

Figure 11. (a) Structure of macrofiber composite (MFC). (b) Shrinkage of MFC upon application of constant voltage. (c) Front and side surfaces of the packaged MFC (Ref. [26], Figure 1). 
Low-coherence ilumination light source

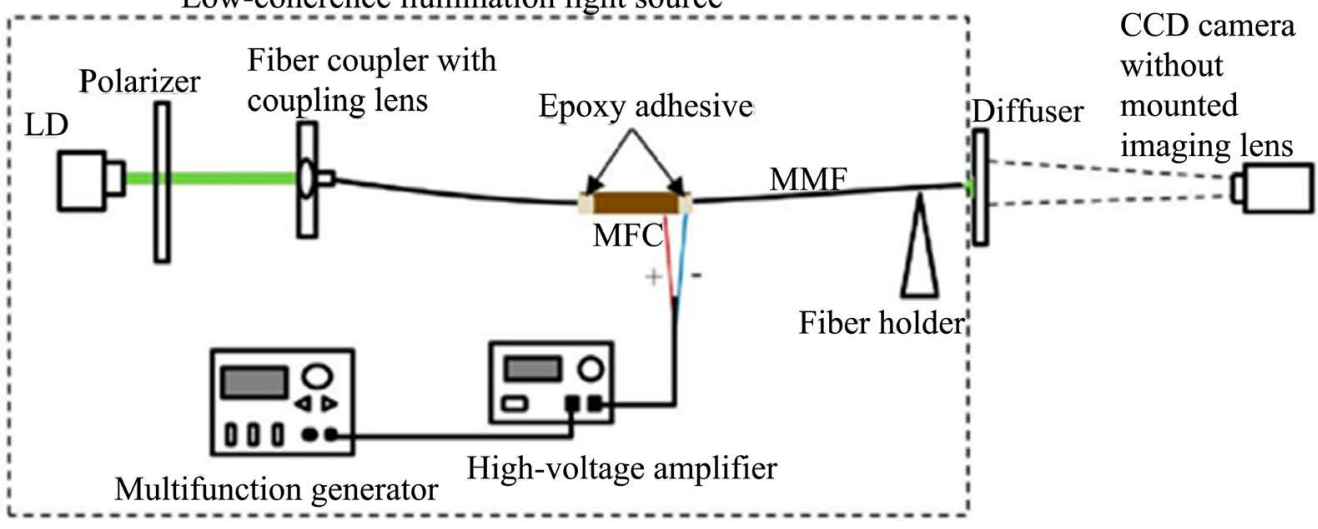

Figure 12. Schematic of an experimental setup with a combination of multimode fiber (MMF) and macrofiber composite (MFC) (Ref. [26], Figure 2).

Speckle pictures are captured by a CCD camera without an imaging lens attached. The CCD camera has a resolution of $1280 \times 1024$ pixels with a pixel size of $5.2 \mu \mathrm{m} \times 5.2 \mu \mathrm{m}$. The multifunction generator generates sinusoidal voltages that are amplified by the high-voltage amplifier for driving the MFC and cause the fiber to oscillate periodically.

The dynamic speckle patterns generated by the MMC vibration were averaged over the exposure time by the CCD video camera or the observer's eye (reaction of $30 \mathrm{~ms}$ ).

The frequency dependences of the speckle contrast for fibers A and B are shown in Figure 13. The excitation voltage was fixed at $100 \mathrm{~V}$. The excitation frequency of the MFC was varied in $10 \mathrm{~Hz}$ intervals in the range from 0 to 200 Hz. Although higher resonant frequencies can also be used, the typical inertia time of the eye is $\sim 30 \mathrm{~ms}$ (corresponding to $33.3 \mathrm{~Hz}$ ), and all speckle patterns created to reduce speckle contrast had to be used within one MFC working period.

The highest efficiency of speckle suppression was achieved at resonance frequencies of about $20 \mathrm{~Hz}$ and about $80 \mathrm{~Hz}$ in the fiber $\mathrm{A}$ and at $60 \mathrm{~Hz}$ and $120 \mathrm{~Hz}$ in the fiber B. To evaluate the efficiency of speckle suppression depending on the control voltage, frequencies of $80 \mathrm{~Hz}$ for the fiber $\mathrm{A}$ and $120 \mathrm{~Hz}$ for the fiber $\mathrm{B}$ were chosen. Experimental dependences of speckle contrast and efficiency of speckle suppression on the control voltage for the fibers A and B are shown in Figure 14.

From the point of view of speckle suppression, the most effective excitation conditions were the following: $80 \mathrm{~Hz}, 150 \mathrm{~V}$ for the fiber A and $120 \mathrm{~Hz}, 150 \mathrm{~V}$ for the fiber B. Under these conditions, the speckle contrast decreased from 0.43 to 0.08 for the fiber $\mathrm{A}$ and from 0.48 to 0.19 for the fiber B. Consequently, the most efficient fiber was the fiber $\mathrm{A}$, which had a higher numerical aperture.

\subsection{Despecklers Displaying Orthogonal Functions}

In [27], a speckle suppression method was described using a space-time phase 

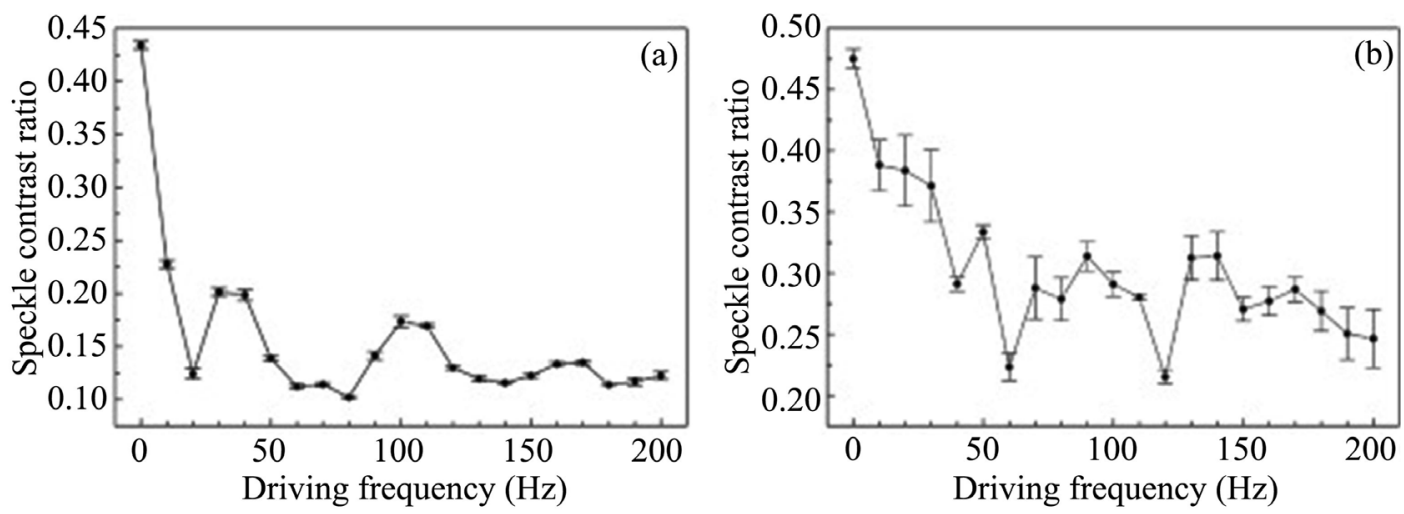

Figure 13. Relationship between the speckle contrast ratio and the excitation frequency of the macrofiber composite (Ref. [26], Figure 3).

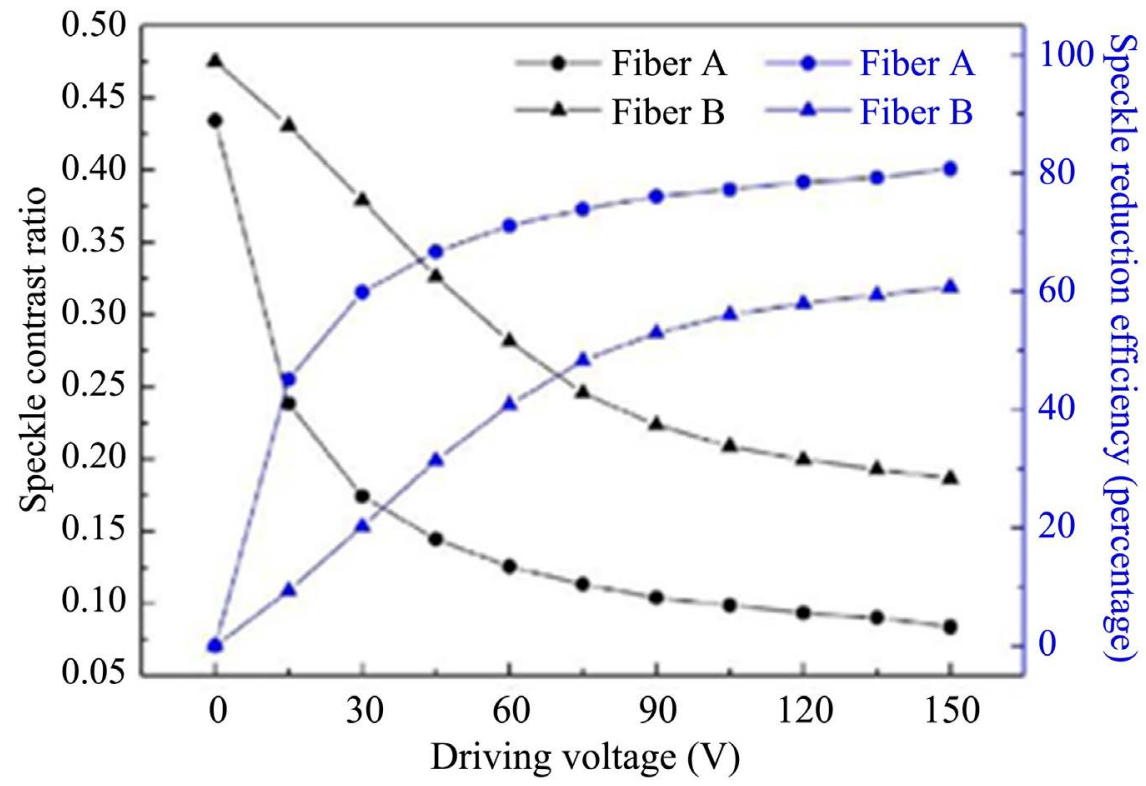

Figure 14. Relationship between speckle contrast (black line), speckle reduction efficiency (blue line), and macrofiber excitation voltage (MFC) for fiber A (circles) and fiber B (triangles) at optimized frequencies (Ref. [26], Figure 4).

light modulator, for example, a liquid crystal one, which is a matrix of separately addressable liquid crystal cells. When controlling the electric voltage using a specially developed computer program, such a spatial light modulator (SLM) in real time can display various orthogonal matrices of phase shift of 0 and $\pi$ corresponding to the Hadamard, Walsh or other orthogonal functions [27] [28] [29].

An example of a phase mask corresponding to the Hadamard matrix is given in [29] [30] and is shown in Figure 15. Here, in the matrix elements (pixels), conventionally painted in black, there is no phase shift, and in the unpainted ones it is equal to $\pi$, or vice versa.

The formation of the Hadamard matrix and the reduction of speckle contrast were demonstrated [31] on a laser projection display with Grating Light Valve 


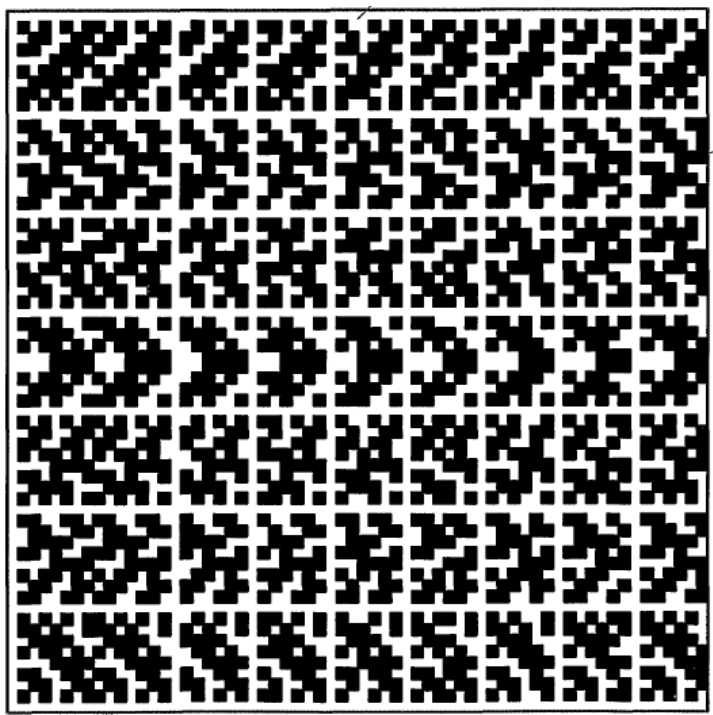

Figure 15. An example of a phase mask corresponding to the Hadamard matrix (Ref. [30], Figure 18).

(GLV) - a one-dimensional spatial light modulator based on MEMS and with a scanner-galvanometer mirror for obtaining a two-dimensional image of $1080 \times$ 1920 pixels on the screen. A diffuser was also used in the intermediate plane of the image to reduce the contrast of speckles. In this configuration, the value of the contrast of speckle structures was reduced to $8 \%$.

The formation of binary phase matrices and their use for suppressing speckles was also tested in [32] [33]. To simplify the fabrication of matrices and control electronics in [13] the two-dimensional binary Hadamard phase matrix was replaced by two one-dimensional binary phase matrices located next to each other. To prove the concept that the effect on light did not change, binary phase codes were etched in the glass. The result of the experiments was a decrease in speckle contrast to a level of $9 \%$.

At present, purely phase modulation of light, which is in great demand in holographic applications, is most easily accomplished in SLMs-transmission type liquid crystal microdisplays using the electro-optical effect of controlled birefringence [34]. For example, the company HOLOEYE [35] offers a GAEA micro-display with a format of $3840 \times 2160$ pixels, with a pixel pitch of $3.74 \mu \mathrm{m}$ and an image change rate of $30 \mathrm{~Hz}$. Phase only SLMs PLUTO and LETO with a format of $1920 \times 1080$ pixels (HD) and a step of 8.0 and $6.4 \mu \mathrm{m}$ respectively have the frequency of $60 \mathrm{~Hz}$. Reflective micro-displays based on the LCoS structure (liquid crystal on a silicon substrate with control microcircuits manufactured in it) have similar parameters in resolution, but a significantly higher (up to 120 $\mathrm{Hz}$ ) frequency of image change.

It should be admitted that the use of an electro-optical medium, divided onto light modulation channels (pixels), with an electronic addressing device for each channel, i.e. in fact, a whole display (microdisplay), strongly complicates and increases in price the optical speckle suppression system. Because of decreasing the 
working aperture (due to the presence of control electronic elements and the gaps between matrix elements) the light losses in such a device are quite large. Moreover, the periodic structure of the matrix introduces distortions of the wave front of coherent light, resulting in distortion of the image on a screen. Electronic control of the spatial light modulator is also a separate complex problem. Therefore, such a despeckler did not receive practical use.

\subsection{Despecklers Using Mirrors}

\subsubsection{Phase-Randomized Deformable Mirror}

Irish company Dyoptyka has developed phase randomized deformable mirror technology to improve illumination uniformity and reduce speckle contrast and other unwanted interference effects that can be observed in laser-illuminated displays [36].

The appearance of a deformable mirror in inactive and active states is shown in Figure 16. To create randomly distributed surface deformations and ensure their consistent behavior over time, an electronic control system based on multilayer piezoceramic elements is used. The deformable mirror substrate can be silicon or glass. The mirror coating is made of silver or dielectric (for higher reflectivity, but its cost increases significantly). Typical deformations of the mirror surface are $\leq 1 \mu \mathrm{m}$ in amplitude) and $\geq 100 \mathrm{kHz}$ in frequency. Speckle contrast is further reduced by the deformable mirror due to angular separation.

For digital cinema projectors, the company has developed deformable mirrors with an active area of about $40 \times 60 \mathrm{~mm}^{2}$, which can withstand up to $100 \mathrm{~W}$ of continuous optical power without destruction. A deformable mirror with an active area of about $3.0 \times 4.5 \mathrm{~mm}^{2}$ and a power consumption of $\leq 30 \mathrm{~mW}$ has been developed for accompanying and built-in pico projectors. Piezoelectric actuators with a nominal service life of 100 thousand hours are used. The $99 \%$ reflectivity in the visible wavelength range was achieved using a dielectric mirror coating.

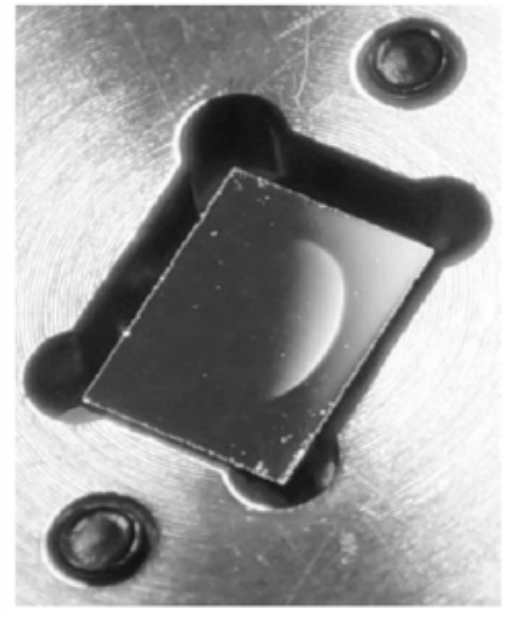

(a)

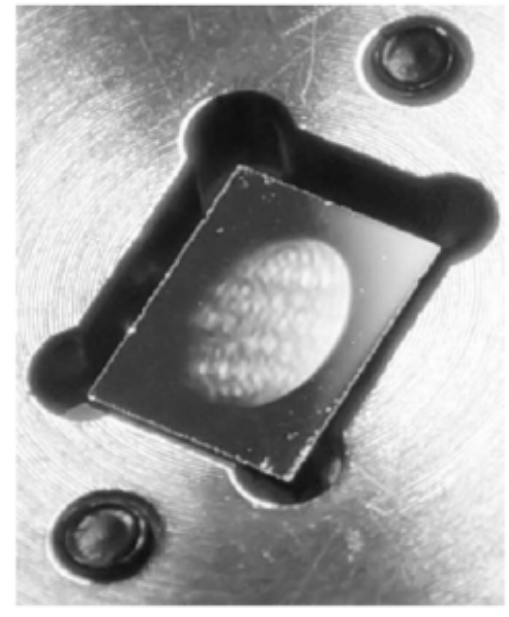

(b)

Figure 16. External view of a deformable mirror in inactive (a) and active (b) states (Ref. [36], Figure 6). 
Surface deformations are within the elastic deformation of the mirror substrate and coatings and do not cause damage. The polarization of the radiation is also conserved.

The main characteristics of the Dyoptyka despeckler are shown in Table 5. This device provides a speckle contrast value of about $3 \%$.

\subsubsection{MEMS-Mirror with Two-Dimensional Beam Deflection}

To create uncorrelated speckle structures due to angular decorrelation, a mirror is used [37], which reflects light beams at different angles (Figure 17). In essence, it is a galvanometer-type microelectromechanical system (MEMS) with a deflected compact and lightweight output mirror. The reflected laser beam hits a random diffuser and is additionally mixed in the "light tube", which results in the appearance of independent speckle patterns, and, as a consequence, suppression of speckles.

A photo of a prototype MEMS mirror with a two-dimensional deflection of the reflected laser beam and its design are shown in Figure 18. Its size is $16 \mathrm{~mm}$ $\times 16 \mathrm{~mm} \times 12 \mathrm{~mm}$. With the help of this device, the speckle contrast value of $4.58 \%$ was achieved.

\subsection{Using Colloidal Dispersion}

In [38], a decrease in speckle-noise is achieved due to the generation of uncorrelated speckle structures when a laser beam passes through a cuvette with a colloidal solution of particles $\mathrm{TiO}_{2}$ (Dupont Ti-Pure R-900, radius $205 \mathrm{~nm}$ ) in a

Table 5. Main characteristics of the Dyoptyka despeckler [36].

\begin{tabular}{cc}
\hline Dimensions, $\mathrm{mm}$ & $3.0 \times 4.5$ \\
\hline Voltage, $\mathrm{V}$ & 5 \\
Power consumption, $\mathrm{mW}$ & 100 \\
Working temperature, ${ }^{\circ} \mathrm{C}$ & $-40 \ldots+85$ \\
Speckle structure contrast, $\mathrm{C}, \%$ & 3 \\
\hline
\end{tabular}

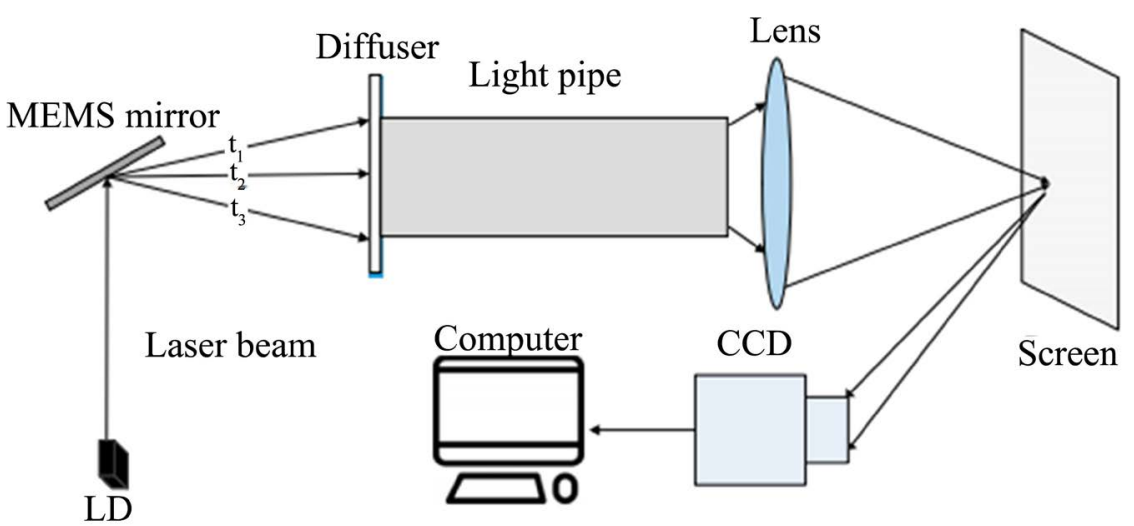

Figure 17. Scheme of operation of a despeckler based on a MEMC mirror (Ref. [37], Figure 8). 


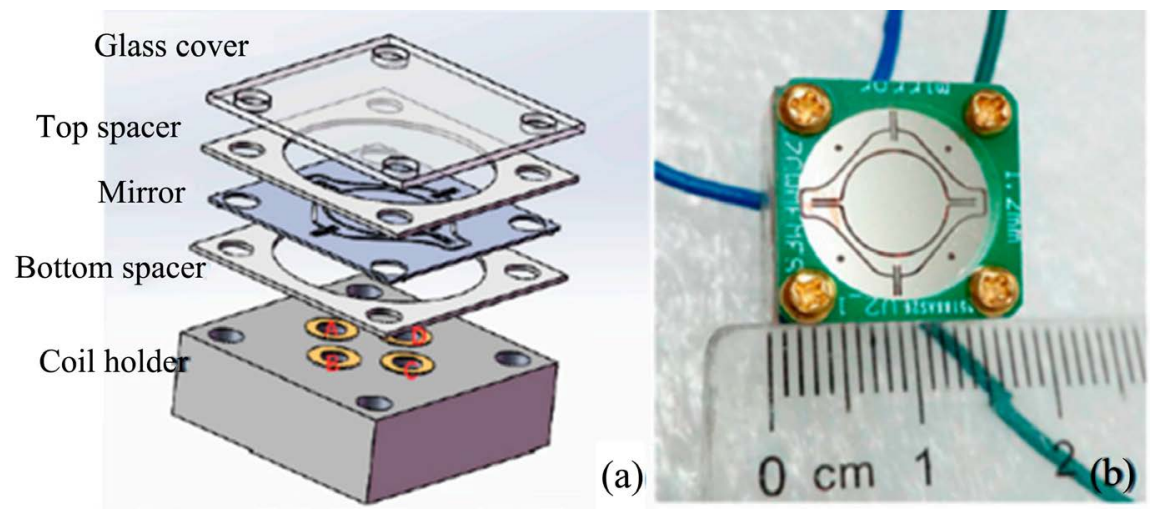

Figure 18. Photo of a prototype two-dimensional MEMS mirror (right) and its components (Ref. [37], Figure 4).

water. The particle density is $2 \times 10^{10} \mathrm{~cm}^{-3}$. Such a solution acts as a diffuser moving without external energy. Really, due to Brownian motion in a solution at room temperature, uncorrelated speckle structures are generated rather quickly (for about $1 \mu \mathrm{s}$ ).

The external view of the setup with the cuvette and its diagram are shown in Figure 19. In order to minimize losses during the propagation of radiation through the colloidal solution, the sides of the cuvette are covered with a thick layer of white paint, which is highly reflective and has little absorption. To deliver a laser beam (wavelength $532 \mathrm{~nm}$ ) deep into the colloidal solution, a single-mode optical fiber is used, providing a transmission of more than $90 \%$.

Speckle contrast measurements are made as follows. The laser beam is input from the free space into the colloidal solution from above, and in general, the fiber tip is placed at a distance $L$ from the cuvette bottom, and the total thickness of the solution in the cuvette is $t$ (Figure 19(d)). The scattered laser radiation is collimated by the objective (Figure 20(a)) and falls on a detector (CCD camera).

With the single-mode fiber tip at the top of the solution, the light is scattered mainly at the top part of the cuvette (Figure 19(a)). When the tip is immersed, radiation is visible in the lower part of the cuvette and on the sides (Figure 19(b)). With deep delivery of radiation into the colloidal solution, a strong radiation output is observed through the bottom of the cuvette (Figure 19(c)). Due to multiple scattering in a colloidal solution, the angular distribution of scattered radiation emitted through the cuvette bottom is similar to the distribution of radiation from a Lambert source.

Speckle patterns were recorded at different distances from the cuvette bottom. Based on the contrast of the speckles, the number of independent speckle patterns and the decorrelation time were determined as a function of $L$ (Figure 20(d), Figure 20(e)). Without colloidal solution, the observed speckle contrast was $C=0.34$. The use of a colloidal solution made it possible to reduce the speckle contrast value to $C=0.032$ at $L=5 \mathrm{~mm}$.

Thus, the Brownian motion of colloidal particles results in both rapid temporal changes in the laser beam and angular diversity, which makes it possible to 


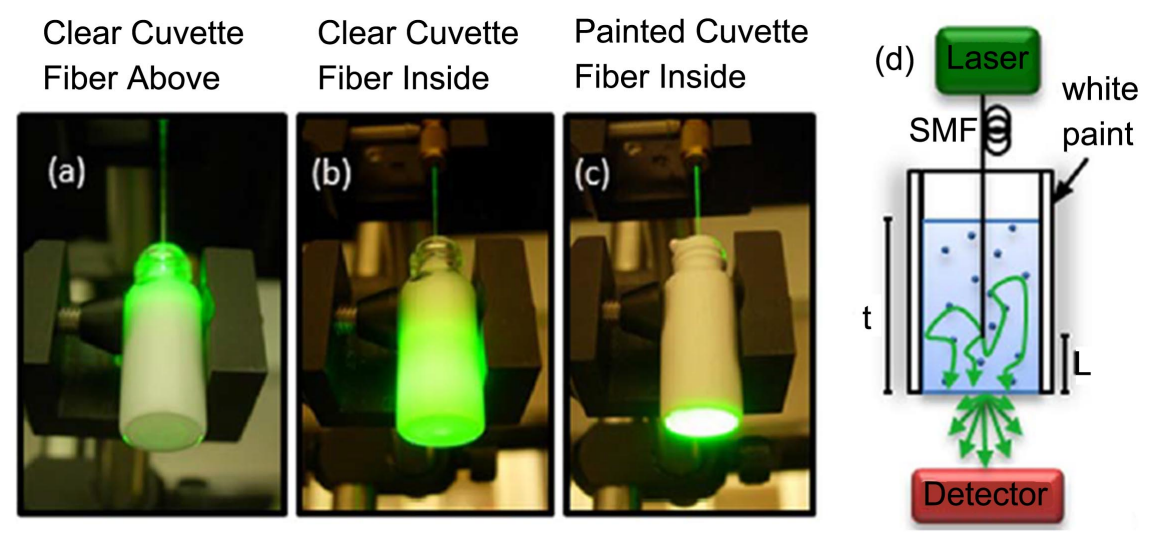

Figure 19. Photographs of a transparent glass cell with a colloidal solution of $\mathrm{TiO}_{2}$ particles in water (a, b, c) and a scheme of the setup operation (d) (Ref. [38], Figure 1).

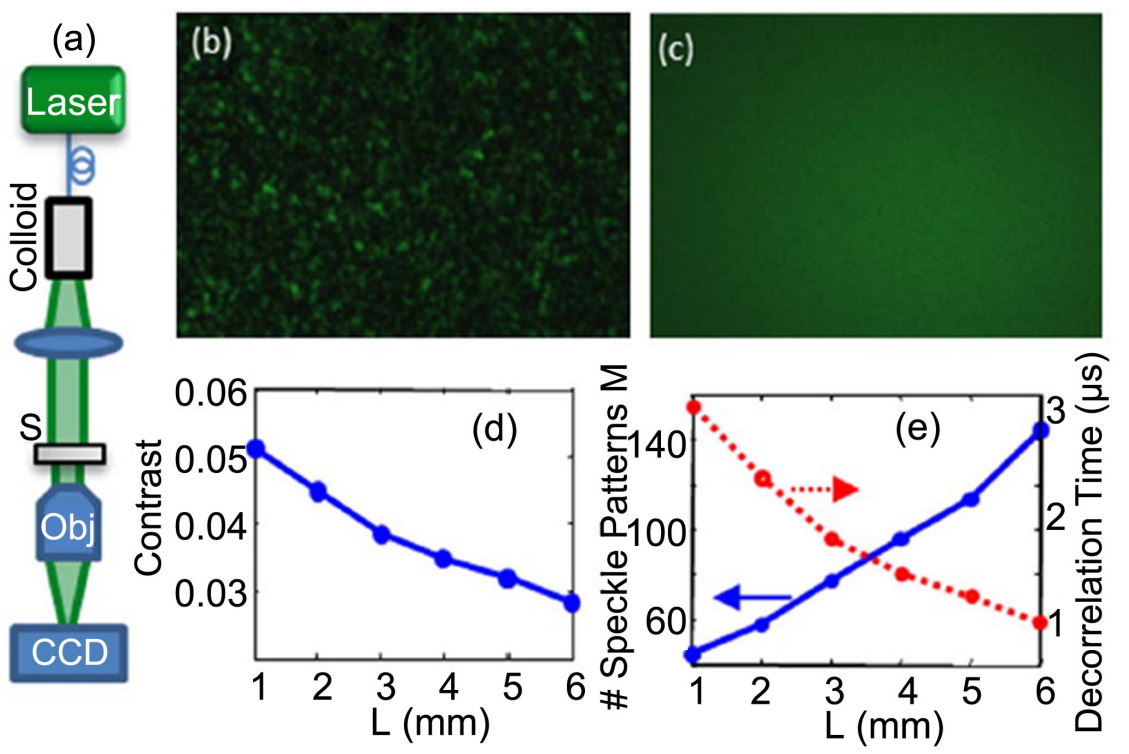

Figure 20 (Ref. [38], Figure 2). (a) Scheme of the experimental setup used to measure speckle contrast; (b) Speckle pattern in the absence of colloidal solution in the cuvette; (c) Speckle pattern in the presence of a colloidal solution in the cuvette; (d) Measured speckle contrast as a function of the distance $L$ between the fiber tip and the cuvette bottom; (e) Number of speckle patterns $M$ versus $L$.

rapidly reduce the speckle contrast to $3.2 \%$. A significant advantage of using a colloidal solution for speckle-noise reducing is that there is no need for external power. However, to ensure stable operation over many years of service, it is necessary to control the chemical composition and electrostatic charge of the particles. In addition, a despeckler based on the use of colloidal solutions is quite difficult to make compact.

\subsection{Despecklers of Electro-0ptical Type}

\subsubsection{Polymer Stabilized Nematic Liquid Crystal (PSLC) Twist Cell}

In the work of Taiwanese scientists [39], a method for suppressing speckle-noise using a liquid crystal (LC) stabilized by a polymer network with a high initial 
optical transmittance was proposed. For this, they used four samples of LC cells with twist molecule orientation. These samples were obtained by mixing nematic LC with photocurable biphenyl methacrylate monomers (OC) of various concentrations. Samples I (a concentration of $3 \mathrm{wt} \%$ OC), II ( $5 \mathrm{wt} \%$ OC), and III (7 wt $\%$ OC) were standard LC cells of $7.5 \mu \mathrm{m}$ thick with transparent ITO electrodes and a rubbed layer polymerized polyimide (orienting layer) on glass plates. In addition to them, there was also a sample IV twist cell (a concentration of $5 \mathrm{wt} \%$ OC) where LC was in a wedge-shaped gap with dimensions of 4.5 and $8 \mu \mathrm{m}$ on different sides of the cell.

A polymer network is required to stabilize and/or blocking LC molecules at different orientations in the layer (with different optical states), as well as to reduce the switching time and operating voltage. When a voltage was applied, a large depth of light modulation was obtained due to light scattering caused by a mismatch between the refractive indices of the reoriented LC layer and the polymer network. In essence, it is an electric field-activated diffuser that is switched off by switching off the voltage.

Figure 21 shows an experimental setup for studying light modulation in PSLC cells, which includes a light source, lenses, a light modulating cell with a voltage generator, and a measuring unit with a spectrometer.

Table 6 for all PSLC cell samples shows the minimum values of transmission and contrast of speckle patterns, maximum values of the modulation depth and the rate of speckle-noise reduction, as well as the applied voltage values corresponding to them.

The results showed (see Figure 22(a) and Table 6) that the highest efficiency

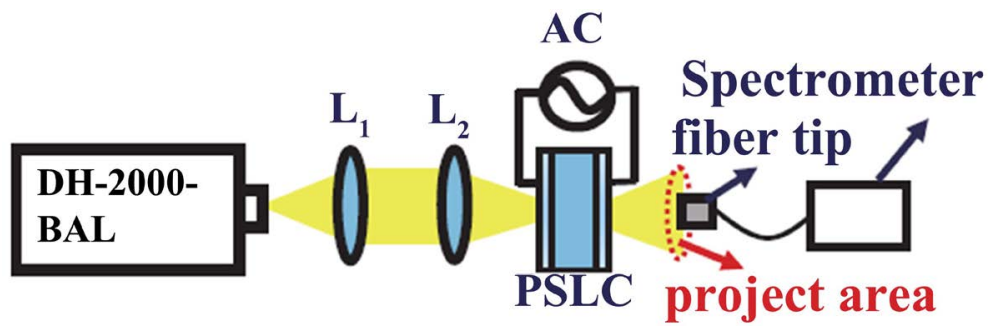

Figure 21. Experimental setup for studying light modulation using a PSLC cell at different values of the control voltage (Ref. [39], Figure 3).

Table 6. Minimum transmission $\left(T_{\min }\right)$, maximum modulation depth $\left(\Delta T_{\max }\right)$, minimum speckle contrast $\left(C_{\min }\right)$, maximum speed of speckle noise reduction $\left(\alpha_{\max }\right)$, voltage providing minimum transmission $\left(V_{a}, T_{\min }\right)$, and voltage providing minimum speckle contrast $\left(V_{a}\right.$ C Cmin $)[39]$.

\begin{tabular}{ccccccc}
\hline Sample & $T_{\min }, \%$ & $\Delta T_{\max }, \%$ & $V_{a}, T_{\min }, V$ & $C_{\min }$ & $\alpha_{\max }, \%$ & $V_{a}, C_{\min }, V$ \\
\hline I & 43.0 & 42.8 & 5 & 0.050 & 47.6 & 3 \\
II & 35.3 & 47.6 & 7 & 0.043 & 54.7 & 6 \\
III & 40.0 & 39.2 & 10 & 0.052 & 54.7 & 6 \\
IV & 38.1 & 44.8 & 10 & 0.025 & 74.7 & 10 \\
\hline
\end{tabular}




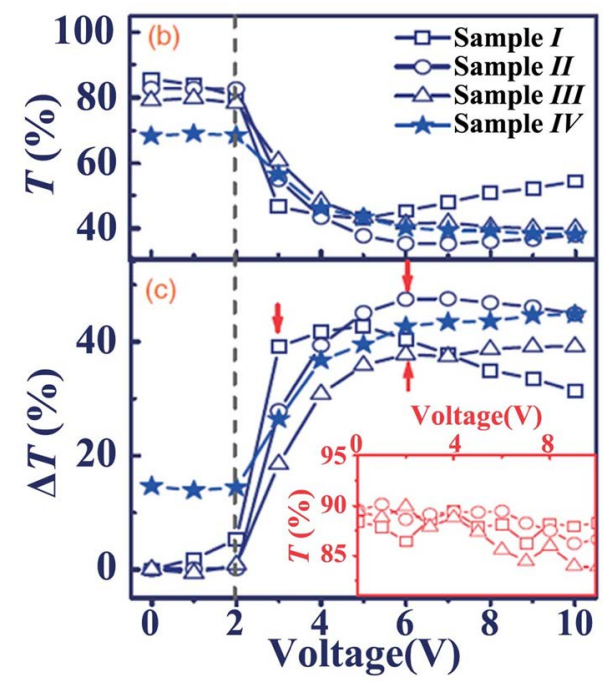

(a)

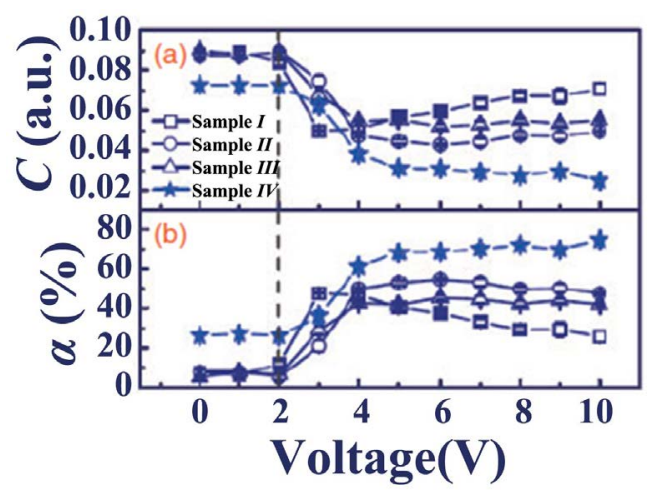

(b)

Figure 22. Dependences of light transmission, modulation depth, contrast of speckle structures and rate of reduction of speckle-noise on the voltage applied to the PSLC cell (Ref. [39], Figures 3, 5): a) Dependences of light transmission (T) and modulation depth $(\Delta T)$; (b) Dependences of the contrast of speckle structures $(C)$ and the rate of reduction of speckle noise $(\alpha)$.

of speckle-noise suppression (with a speckle contrast value of about 0.025 ) at low applied voltage values $(\leq 10 \mathrm{~V})$ was achieved in a wedge-shaped LC cell.

Paper [39] does not provide information on the mass-dimensional parameters of an experimental despeckler based on a PSLC cell. However, taking into account the current level of development of LCD-based devices and the characteristics of the LCD despeckler described in the next section, it can be assumed that such a despeckler is rather compact. The disadvantage is the limited temperature range $\left(0 \ldots+60^{\circ} \mathrm{C}\right)$.

\subsubsection{Electro-Optical Cell with a Ferroelectric LC}

To suppress speckle-noise at the P.N. Lebedev Physical Institute a method was proposed using an electro-optical cell with a ferroelectric liquid crystal (FLC), initially with a helical (spiral) structure [40] [41], and then with a helix-free structure [42] [43] [44], when the original helical structure was suppressed com- 
pletely by introducing chiral impurities possessing opposite sign of optical activity.

In both types of FLC cells effective suppression of speckle-noise was, when specially formed bipolar two-frequency electric pulses were applied. Short-term application of an electric field to the FLC resulted in the formation of a structure of transition domains-spontaneously ordered regions, at the boundaries of which light scattering occurs. As a result of light scattering in the bulk of the FLC layer, structures with a random distribution of refractive index gradients were formed, which are the cause of spatially inhomogeneous (over the beam cross section) phase modulation of a laser beam and phase relation destroy in it [40]. Note that short-term light scattering with a duration of less than $50 \mu$ s is not sensitive to the eyes, does not distort the structure of images, does not affect their perception, and light losses are insignificant (less than 5\%).

Relative to FLC with helix structure, helix-free FLC provided such advantages [42] as the absence of distortions in the spectral composition of modulated light radiation and the absence of light scattering when the electric field is turned off. In addition, in helix-free FLC the effect of spatially inhomogeneous phase modulation of light was much less inertial.

In the despeckler prototype with a helix-free FLC the following material parameters were: spontaneous polarization $P_{S}=40 \mathrm{nC} / \mathrm{cm}^{2}$, rotational viscosity $\gamma_{\varphi}$ $=0.7$ Poise, tilt angle of molecules in smectic layers $\Theta_{0}=23^{\circ}$ (at the temperature of $20^{\circ} \mathrm{C}$ ), temperature range of existence of ferroelectric (chiral smectic $\mathrm{C}^{\star}$ ) phase from $2^{\circ} \mathrm{C}$ to $75^{\circ} \mathrm{C}$ [43]. The thickness of electro-optical cells varied from 10 to 20 microns. Increasing the layer thickness to 18 - 20 microns and choosing a control mode with a bipolar pulse duration corresponding to different maxima of light scattering [44], allowed not only to reduce the contrast of speckle structures to 0.07 (Figure 23), but also to increase the modulation frequency of the phase delay to $2 \mathrm{kHz}$ (with an increase in the control voltage to $60-65 \mathrm{~V}$ ).

A higher value of speckle contrast when using a helix-free FLC may be due to less accurate settings of the measurement parameters. It is important that a well-developed technology makes it possible to vary the size of the FLC despeckler aperture from fractions to several square centimeters with a thickness of 1 - 2 $\mathrm{mm}$, including the control chip. Unfortunately, the despeckler, like all LCD devices, has limitations in the operating temperature range (approximately from zero to $\left.+60^{\circ} \mathrm{C}\right)$.

\subsection{Despecklers Based on the Use of Diffraction Gratings}

In a few works the possibility of using diffraction gratings for speckle averaging was investigated [7]. Obtained results on the reduction of speckle contrast and other characteristics cannot be classified as practically significant, but the methods used are interesting and will be briefly described below.

In [45], dynamic diffraction gratings were made of an electroactive polymer that could be electrostatically activated. When light diffracted on several gratings 


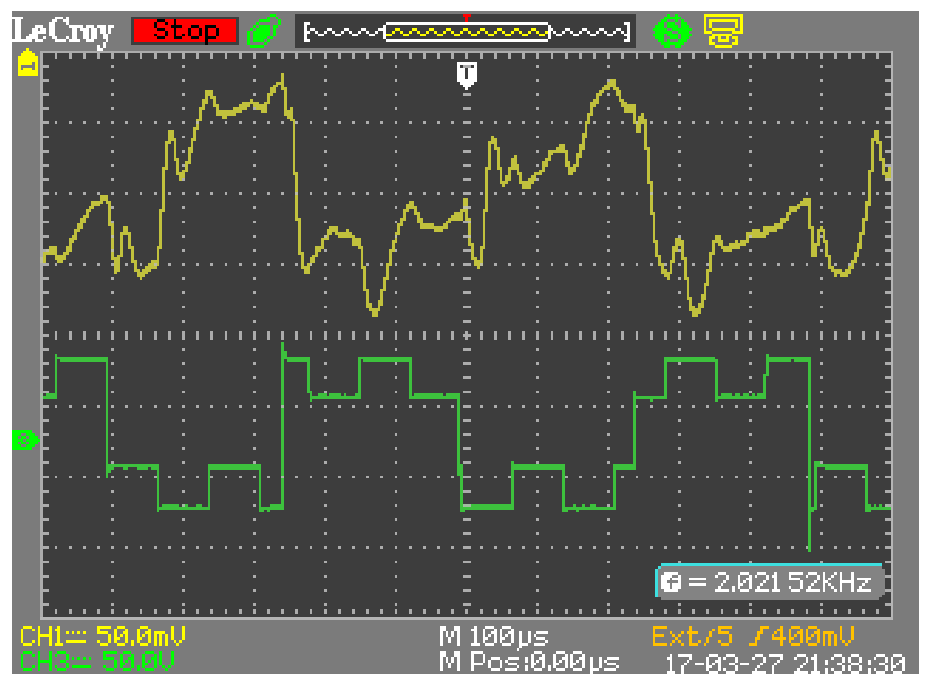

(a)

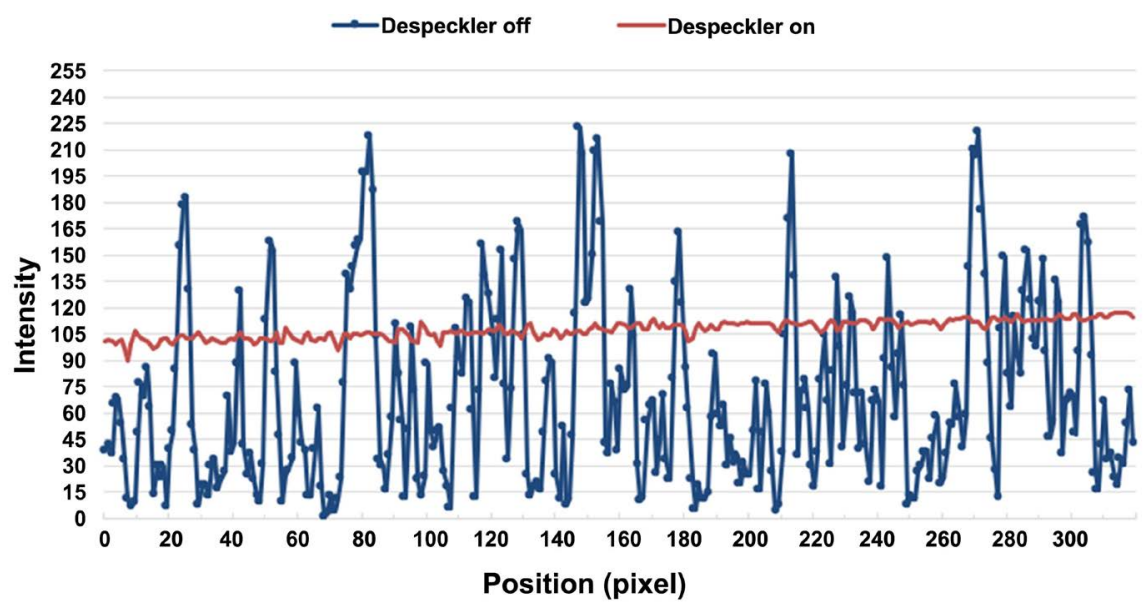

(b)

Figure 23. Control voltage, modulation of the phase delay and distribution of radiation intensity in the cross section of the laser beam behind the FLC cell in the absence of a control voltage at the cell electrodes (despeckler is off) and in the presence (despeckler is on) (Ref. [44], Figure 11): (a) Oscillograms of the control voltage (lower) and phase delay modulation; (b) The distribution of the radiation intensity in the cross section of the laser beam behind the FLC-cell.

of different frequencies (Figure 24), independent speckle patterns of different diffraction orders varying in time on angle and space, were averaged on the CCD camera during its integration time. The resulting speckle contrast of 0.37 was limited because of insufficient interaction length of the electric field and the medium, due to large light losses.

In [46] the method for suppressing speckle structures was demonstrated based on using a relief light modulator (RLM) for fast generation of a sequence of independent dynamic diffraction gratings. Technically, RLM as a speckle suppressor is an assembly of two dielectric plates with a gas gap, which should not be less than the nano-relief created on the surface of an elastic-viscous (gel) layer (Figure 25). 


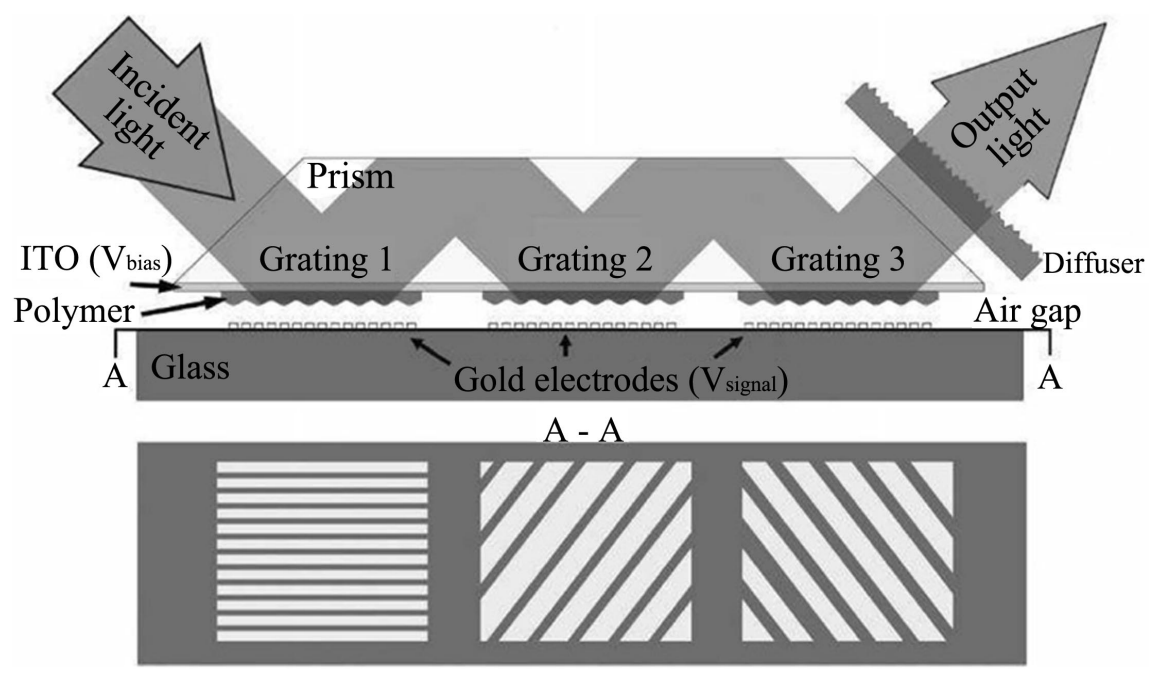

Figure 24. Side and top views of polymer diffraction gratings with three types of electrodes (Ref. [7], Figure 3).

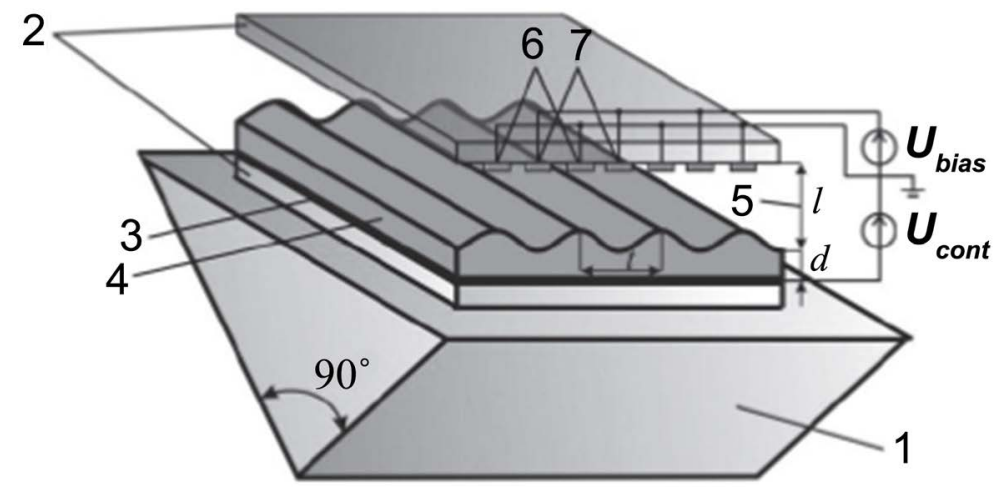

Figure 25. Scheme of the Relief Light Modulator (Ref. [46], Figure 1): 1-glass prism; 2-glass substrates; 3-solid electrode; 4-dielectric deformable gel-like layer of thickness $d$ with dielectric constant $\varepsilon 1 ; 5$-air gap of width 1 with dielectric constant $\varepsilon 2$; 6-grounding electrodes; 7-control electrodes; $U_{\text {bias }}-$ constant bias voltage; $U_{\text {cont }}-$ control voltage; $t$ is the spatial period of the relief.

Modulation of light is carried out due to the diffractive nano-relief created on the surface of a thin transparent dielectric layer at the appearance of ponderomotive forces in it when an electric voltage is applied. In the case when there is no electric voltage on electrodes, the gel-like layer does not change its shape (remains flat) and all incident rays are reflected from it as from a flat mirror. When a control signal is applied, a sinusoidal grating is formed on the gel-like layer, and the rays are diffracted on it according to the light diffraction law. Unfortunately, this type of despeckler also has large light losses and cannot be compact due to operating the diffraction grating only for reflection. In addition, the Doppler wavelength change and fatigue of the grating material are noticeable in the RLM.

Problems can also be foreseen when using another polymer medium, not dynamic and thin, but bulk (the thickness is significantly greater than the wave- 
length), in which many diffraction gratings with different wave vectors are formed not electrically, but by light. A device based on a photopolymer medium is proposed for speckle suppression in [47]. This medium changes the refractive index when illuminated by a laser beam. By means of a deflecting device and lenses forming laser beams converging and diverging at an arbitrary angle, numerous speckle images are recorded in the medium in the form of superimposed phase holograms. According to the associativity property of holograms, when the laser beam passes through the photopolymer medium, it is automatically "cleaned" of speckles.

Such a despeckler (beam shaper-beam corrector, as the authors called it) does not require control and can even serve as a matrix for replicating new products by pressing. However, it also has significant drawbacks: introducing additional noise from superimposed holograms; the criticality of speckle contrast to the exact compliance of the wavelength and angle of beam incidence; difficulties in creating a compact device and the problem of "fatigue" of the photopolymer medium during operation.

\section{Conclusions}

The search for methods of suppressing speckles in recent years has become especially relevant in connection with works of leading electronic companies (LG, Samsung, etc.) that develop the holographic displays and laser video projectors, both large-screen theatrical and compact pico-projectors placed inside a mobile phone and projecting video images (including television) onto a desktop or wall screen.

We considered above many methods and devices, which can effectively suppress speckle-noise and provide thereby for the observer an acceptable quality of images formed by a laser (with speckle contrast $\leq 10 \%$ ). In particularly there were analyzed a vibrating diffuser; an electroactive polymer (elastic membrane) moving the diffuser; an optical fiber deformed by a piezoelectric vibrator; a mirror with phase randomization deformed by piezoceramic elements; a micro-mirror deflected in an electromechanical system; polymer and photopolymer medium; a colloidal solution with dispersed particles. As well we considered possible use of electro-optical-liquid crystal despeckers based on a light-scattering twist cell with a nematic LC stabilized by a polymer network and based on a cell with the ferroelectric LC implementing spatially inhomogeneous phase modulation of light.

The main characteristics of the described despecker developments for their comparison and assessing are summarized in Table 7. The number of crosses qualitatively indicates the value of the considered characteristic for a particular despekler. Fewer crosses along with less contrast magnitude are obviously preferable. However, it should be taken into account also that in different applications of despeckers, the significance of one or another characteristic may differ essentially. 
Table 7. Characteristics of the developed despeklers.

\begin{tabular}{|c|c|c|c|c|c|c|c|}
\hline \multirow{2}{*}{$\begin{array}{l}\text { Operation mechanism and } \\
\text { type of despeckler }\end{array}$} & \multicolumn{3}{|c|}{ Light scattering } & \multicolumn{2}{|r|}{ Fhase } & \multirow{3}{*}{$\begin{array}{l}\text { Combined } \\
\text { EO cell with } \\
\text { helix-free LC }\end{array}$} & \multirow{3}{*}{$\begin{array}{l}\text { Light deflection } \\
\text { MEMS mirror }\end{array}$} \\
\hline & \multirow{2}{*}{$\begin{array}{l}\text { Shifting diffuser } \\
\text { LSR-5-17 }\end{array}$} & \multirow{2}{*}{$\begin{array}{c}\text { Switch-on } \\
\text { diffuser PSLC }\end{array}$} & \multirow{2}{*}{$\begin{array}{l}\text { Colloidal } \\
\text { solution }\end{array}$} & \multirow{2}{*}{$\begin{array}{l}\text { Opticalfiber } \\
\text { and vibrator }\end{array}$} & \multirow{2}{*}{$\begin{array}{l}\text { Deformable mirror } \\
\text { Dyoptyka }\end{array}$} & & \\
\hline Characteristics: & & & & & & & \\
\hline Dimensions and weight & $\mathrm{xx}$ & $\mathrm{x}$ & $\mathrm{xxx}$ & $\mathrm{xx}$ & $\mathrm{xx}$ & $\mathrm{x}$ & $\mathrm{x}$ \\
\hline Power consumption & $\mathrm{xx}$ & $\mathrm{x}$ & - & $\mathrm{xx}$ & $\mathrm{xx}$ & $\mathrm{x}$ & $\mathrm{x}$ \\
\hline Light losses & $\mathrm{xx}$ & $\mathrm{xx}$ & $\mathrm{xx}$ & $\mathrm{x}$ & $\mathrm{x}$ & $\mathrm{x}$ & $\mathrm{xx}$ \\
\hline Complexity of electronics & $\mathrm{xx}$ & $\mathrm{x}$ & - & $\mathrm{xx}$ & $\mathrm{xx}$ & $\mathrm{xx}$ & $\mathrm{xx}$ \\
\hline Complexity of optics & $\mathrm{xxx}$ & $\mathrm{xxx}$ & $\mathrm{xxx}$ & $\mathrm{xx}$ & $\mathrm{xx}$ & $\mathrm{x}$ & $\mathrm{xx}$ \\
\hline Temperature requirements & - & $\mathrm{xxx}$ & $\mathrm{xx}$ & - & $\mathrm{x}$ & $\mathrm{xxx}$ & $\mathrm{x}$ \\
\hline Difficulties in commercializ. & $\mathrm{x}$ & $\mathrm{xx}$ & $\mathrm{xxx}$ & $\mathrm{xx}$ & $\mathrm{x}$ & $\mathrm{xx}$ & $\mathrm{xx}$ \\
\hline Speckle contrast value, $\%$ & 2 & 2.5 & 3.4 & 5.6 & 3 & 7 & 4.6 \\
\hline
\end{tabular}

The data in Table 7 indicate great progress in the development of efficient despeckers. Note that despeklers have advantages that do not have mechanically deformable or movable elements usually complicating the design, increasing dimensions and reducing the reliability and durability of operation.

Of the considered despeckers with high efficiency of speckle-noise suppression, only devices from the companies Optotune (a diffuser on an electroactive polymer) and Dyoptyka (phase deformable mirror) have been brought to commercial use. Their speckle contrast values are $2 \%$ and $3 \%$, respectively; these devices are compact, have low light losses, and they have no problems with operating temperature.

The developments of devices for suppressing speckle-noise based on the deflected mirrors and optical fibers are also effective. Despekers based on LCD cells have the same indicators. They also have low power consumption, low light losses and are compact, but they have limitations in temperature range. There is an interesting device based on a colloidal solution with dispersed particles, which does not require external energy and, accordingly, electronics for control at all, but it is difficult to make it compact and stable for a long time.

Table 7 does not contains speckle suppression devices by means of phase matrices implementing orthogonal functions and diffraction gratings based on polymer and photopolymer media, since their further development was not. Nevertheless, their description in the review is given specially for indication of the variety of the proposed physical methods and their development.

The problem of obtaining speckle-free images formed by a laser beam has been around for about 40 years. However, the data presented in the review indicate that this problem cannot be considered solved, and in the future one can expect the appearance of other methods and new materials and devices for speckle suppression.

\section{Funding}

This work was carried out within the framework of the Russia State Assignment 
No. 0023-2019-0002.

\section{Conflicts of Interest}

The authors declare no conflicts of interest.

\section{References}

[1] Goodman, J.W. (1976) Some Fundamental Properties of Speckle. Journal of the Optical Society of America, 66, 1145-1150. https://doi.org/10.1364/JOSA.66.001145

[2] Lowenthal, S. and Joyeux, D. (1971) Speckle Removal by a Slowly Moving Diffuser Associated with a Motionless Diffuser. Journal of the Optical Society of America, 61, 847-851. https://doi.org/10.1364/JOSA.61.000847

[3] Turukhano, B.G. (1985) Circular Holographic Memory System. Proceedings of Optical Holography Practical Applications, 75-95.

[4] Goodman, J.W. (1984) Laser Speckle and Related Phenomena. Springer-Verlag, Berlin, 9-75.

[5] Roelandt, S., Meuret, Y., Jacobs, A., Willaert, K., Janssens, P., Thienpont, H. and Verschaffelt, G. (2014) Human Speckle Perception Threshold for Still Images from a Laser Projection System. Optics Express, 22, 23965-23979.

https://doi.org/10.1364/OE.22.023965

[6] Verschaffelt, G., Roelandt, S., Meuret, Y., Van den Broeck, W., Kilpi, K., Lievens, B., Jacobs, A., Janssens, P. and Thienpont, H. (2016) Speckle Perception and Disturbance Limit in Laser Based Projectors. Proceedings of SPIE Optics, Photonics and Digital Technologies for Imaging Applications IV, 9896, 9896R. https://doi.org/10.1117/12.2227535

[7] Akram, M. and Chen, X. (2015) Speckle Reduction Methods in Laser-Based Picture Projectors. Optical Review, 23, 108-120. https://doi.org/10.1007/s10043-015-0158-6

[8] Collier, R.J., Burckhardt, Ch.B. and Lin, L.H. (1971) Optical Holography. Academic Press, New York.

[9] Evtikhiev, N.N., Evtikhieva, O.A., Kompanets, I.N., Krasnov, A.E., Kulchin, Yu.N., Odinokov, S.B. and Rinkevichus, B.S. (2000) Information Optics. MPEI Publishing House, Moscow.

[10] Ryabukho, V.P. (2001) Speckle-Interferometry. Soros Educational Journal, 7, 102-109.

[11] Ulyanov, S.S. (1999) What Are Speckles. Soros Educational Journal, 5, 112-116.

[12] Korolenko, P.V. and Maganova, M.S. (2010) Fundamentals of Statistical Methods in Optics. Universitetskaya Kniga, Moscow, 164.

[13] Chellappan, K., Erden, E. and Urey, H. (2010) Laser-Based Displays: A Review. Applied Optics, 49, F79-F98. https://doi.org/10.1364/AO.49.000F79

[14] Goodman, J.W. (2006) Speckle Phenomena in Optics: Theory and Applications. Roberts and Company Publishers, Colorado.

[15] Schmidt, T.C. (2014) Standardization of Speckle Measurement for Large Screen Laser Illuminated Video Projection Systems. Journal of the Society for Information Display, 45, 423-426. https://doi.org/10.1002/j.2168-0159.2014.tb00114.x

[16] Faulhaber, A., Haberl, S., Haist, T., Gronle, M. and Baroud, Y. (2018) Dynamic Holography for Speckle-Noise Reduction in Hybrid Measurement System. Proceedings of SPIE “Laser Beam Shaping XVIIP”, 10744, 107440J-1-107440J-12. https://doi.org/10.1117/12.2320486 
[17] Riechert, F. (2009) Speckle Reduction in Projection Systems. Dissertation, Karlsruhe Institute of Technology, Karlsruhe.

[18] Li, J. (2013) Design of Optical Engine for LCOS Laser Display with Rotated Diffuser Plate. Microwave and Optical Technology Letters, 55, 138-141. https://doi.org/10.1002/mop.27221

[19] Sun, M. and Lu, Z. (2010) Speckle Suppression with a Rotating Light Pipe. Optical Engineering, 49, 024202. https://doi.org/10.1117/1.3314310

[20] Li, D., Kelly, D.P. and Sheridan, J.T. (2013) Speckle Suppression by Doubly Scattering Systems. Applied Optics, 52, 8617-8626. https://doi.org/10.1364/AO.52.008617

[21] http://www.powertechnology.com/

[22] Kubota, S. and Goodman, J. (2010) Very Efficient Speckle Contrast Reduction Realized by Moving Diffuser Device. Applied Optics, 49, 4385-4391.

https://doi.org/10.1364/AO.49.004385

[23] Giger, J., Blum, M. and Aschwanden, M. (2012) Laser Speckle Reduction Based on Electroactive Polymers. The 1st Advanced Lasers and Photon Sources (ALPS 12), Yokohama, 26-27 April 2012.

[24] Georgiades, P., Allan, V.J., Dickinson, M. and Waight, T. (2016) Reducing Laser Speckle with Electroactive Polymer Actuators. Journal of Microscopy, 264, 375-383. https://doi.org/10.1111/jmi.12453

[25] Lin, Y., Fujimaki, Y. and Taniguchi, H. (2014) Reduction of Speckle Contrast in Multimode Fibers Using Piezoelectric Vibrator. Proceedings of SPIE "Laser Resonators, Microresonators, and Beam Control XVP”, 8960, 89601S-1-89601S-7. https://doi.org/10.1117/12.2037431

[26] Gao, Q., Tong, Z., Ma, Y., Wang, M., Jia, S. and Chen, X. (2010) Flexible and Lightweight Speckle-Noise Suppression Module Based on Generation of Dynamic Speckles with Multimode Fiber and Macro Fiber Composite. Optics and Laser Technology, 123, 105941. https://doi.org/10.1016/j.optlastec.2019.105941

[27] Vasiliev, A.A., Kasasent, D., Kompanets, I.N. and Parfenov, A.V. (1987) Spatial Light Modulators. Radio \& Svyaz, Moscow.

[28] Hedayat, A.S., Sloan, N.J.A. and Stufken, J. (1999) Orthogonal Arrays: Theory and Applications. Springer-Verlag, New York. https://doi.org/10.1007/978-1-4612-1478-6

[29] Trisnadi, J.I. (2004) Hadamard Speckle Contrast Reduction. Optics Letters, 29, 11-13. https://doi.org/10.1364/OL.29.000011

[30] Trisnadi, J.I. (2003) Method, Apparatus, and Diffuser for Reducing Laser Speckle. Patent WO 03/001281 A1.

[31] Trisnadi, J.I. (2002) Speckle Contrast Reduction in Laser Projection Displays. Proceedings of SPIE “Projection Displays VIIP”, 4657, 131-137.

https://doi.org/10.1117/12.463781

[32] Akram, M., Kartashov, V. and Tong, Z. (2010) Speckle Reduction in Linescan Laser Projectors Using Binary Phase Codes. Optics Letters, 35, 444-446.

https://doi.org/10.1364/OL.35.000444

[33] Gao, W., Tong, Z. Kartashov, V., Akram, M. and Chen, X. (2012) Replacing Two-Dimensional Binary Phase Matrix by a Pair of One Dimensional Dynamic Phase Matrices for Laser Speckle Reduction. Journal of Display Technology, 8, 291-295. https://doi.org/10.1109/JDT.2011.2181333

[34] Kompanets, I.N. and Andreev, A.L. (2017) Micro-Displays in Spatial Light Modula- 
tion Systems. Quantum Electronics, 47, 294-302. https://doi.org/10.1070/QEL16293

[35] http://holoeye.com/spatial-light-modulators

[36] Shevlin, F. (2018) Phase Randomization for Spatio-Temporal Averaging of Unwanted Interference Effects Arising from Coherence. Applied Optics, 57, E6-E10. https://doi.org/10.1364/AO.57.0000E6

[37] Li, F., Zhou, P., Wang, T., He, J. Yu, H. and Shen, W.A. (2017) Large-Size MEMS Scanning Mirror for Speckle Reduction Application. Micromachines, 8, 140. https://doi.org/10.3390/mi8050140

[38] Redding, B., Allen, G., Dufresne, E.R. and Cao, H. (2013) Low-Loss High-Speed Speckle Reduction Using a Colloidal Dispersion. Applied Optics, 52, 1168-1172. https://doi.org/10.1364/AO.52.001168

[39] Lin, J.-H., Chang, S.-C., Li, Y.-H., Chien, C.-Y., Chen, C.-H., Lin, Y.-C., Wu, J.-J., Tsay, S.-Y. and Chen, Y.-H. (2017) Investigation of Laser Speckle-Noise Suppression by Using Polymer-Stabilized Liquid Crystals within Twisted Nematic Cell. Applied Physics Express, 10, 031701. https://doi.org/10.7567/APEX.10.031701

[40] Andreev, A.L., Kompanets, I.N., Minchenko, M.V., Pozhidaev, E.P. and Andreeva, T.B. (2008) Suppression of Speckle-Noise Using a Liquid Crystal Cell. Quantum Electronics, 38, 1166-1170. https://doi.org/10.1070/QE2008v038n12ABEH013894

[41] Andreev, A., Andreeva, T., Kompanets, I., Minchenko, M. and Pozhidaev, E. (2009) Speckle-Noise Suppression Due to a Single Ferroelectric Liquid Crystal Cell. Journal of the Society for Information Display, 17, 801-807.

[42] Andreev, A., Andreeva, T., Kompanets, I. and Zalyapin, N. (2014) Speckle-Noise Suppression Using a Helix-Free Ferroelectric Liquid Crystal Cell. Quantum Electronics, 44, 1136-1140. https://doi.org/10.1070/QE2014v044n12ABEH015587

[43] Andreev, A., Zalyapin, N., Andreeva, T. and Kompanets, I. (2017) Electro-Optic Despeckler Based on a Helix-Free Ferroelectric Liquid Crystal. Quantum Electronics, 47, 1064-1068. https://doi.org/10.1070/QEL16497

[44] Andreev, A.L., Andreeva, T.B., Kompanets, I.N. and Zalyapin, N.V. (2018) Space-Inhomogeneous Phase Modulation of Laser Radiation in an Electro-Optical Ferroelectric Liquid Crystal Cell for Suppressing Speckle-Noise. Applied Optics, 57, 1331-1337. https://doi.org/10.1364/AO.57.001331

[45] Taganov, A.O., Gushcho, Yu.P. and Dergunov, N.I. (2014) Relief Light Modulator for Eliminating Speckle-Noise. INTERMATIC-2014, 4, 84-87.

[46] Ouyang, G., Tong, Z. Akram, M., Wang, K., Kartashov, V., Yan, X. and Chen, X. (2010) Speckle Reduction Using a Motionless Diffractive Optical Element. Optics Letters, 35, 2852-2854. https://doi.org/10.1364/OL.35.002852

[47] Kazutoshi, I., Kurashige, M., Takanokura, T. and Ohyagi, Ya. (2010) Despeckling Method with Variable Speckle Generator Utilizing Photopolymer Film. Proceedings of the International Display Workshops (IDW), 1471-1474. 